\title{
Determinantes del potencial de desarrollo económico de los municipios de Sevilla 2007- 2012
}

\author{
PÉREZ CONDE, JOSÉ MARÍA \\ Centro Universitario de Osuna \\ Correo electrónico: jmperez@euosuna.org \\ ChaVARria ORTIZ, Carlos \\ Centro Universitario de Osuna \\ Correo electrónico: cchavarria@euosuna.org \\ MORÁN-ÁlVAREZ, JUAN CARLOS \\ Universidad de Sevilla \\ Correo electrónico: jcmoran@us.es
}

\begin{abstract}
RESUMEN
El objetivo general del estudio es cuantificar el potencial económico de 98 municipios de la provincia de Sevilla en los años 2007 y 2012 a partir de una investigación empírica mediante la aplicación del modelo de Rasch. Ello nos permitirá diseñar una estructura o marco analítico genérico de referencia para la planificación y ejecución de las políticas de desarrollo territorial. Podremos ver qué municipios han experimentado una mejora en su Potencial Económico en esta etapa 2007-2012 de recesión económica impactante, así como los municipios que han mostrado un retroceso en este potencial. Además, se delimitará el impacto de cada uno de los factores de potencialidad de desarrollo económico en esta Medida del Potencial Económico (MPE), determinando cómo en ambos años se da una concurrencia tanto en los ítems de alta relevancia (ingresos presupuestarios por habitante y las rentas netas de trabajo por población en edad de trabajar) como en los menos relevantes de cara a incrementar su capacidad para crecer y desarrollarse dado que su consecución es de fácil logro (diversificación productiva y el número de declaraciones por población en edad de trabajar). Todo ello permitirá realizar una planificación más eficiente de las medidas de política económica destinadas a propiciar y fomentar el desarrollo económico de los municipios considerados.

Palabras clave: Modelo de Rasch, potencial de desarrollo económico, municipios, factores de potencialidad, política de desarrollo local.
\end{abstract}

Clasificación JEL: C88; I31; O10; O21; R58.

MSC2010: 62P25. 


\title{
Determinants of the potential economic development of the municipalities of Sevilla 2007-2012
}

\begin{abstract}
The general objective of the study is to quantify the economic potential of 98 municipalities in the province of Seville in 2007 and 2012 from empirical research based on the application of the Rasch model. This will allow us to design a generic analytical framework or frame of reference for the planning and execution of territorial development policies. We can see which municipalities have experienced an improvement in their Economic Potential in this 2007-2012 stage of shocking economic recession as well as the municipalities that have shown a decline in this potential. In addition, the impact of each of the economic development potential factors in this Measure of Economic Potential will be delimited, determining how in both years there is a concurrence in the items of high relevance (budgetary income per inhabitant and the net work-earned income per working-age population) and in those which are least relevant to increase their capacity to grow and develop since their achievement is easy to achieve (productive diversification and the number of declarations per workingage population). All this will allow for more efficient planning of economic policy measures aimed at promoting and fostering the economic development of the municipalities considered.
\end{abstract}

Keywords: Rasch model, potential for economic development, municipalities, potentiality factors, local development policy.

JEL classification: C88; I31; O10; O21; R58.

MSC2010: 62P25.

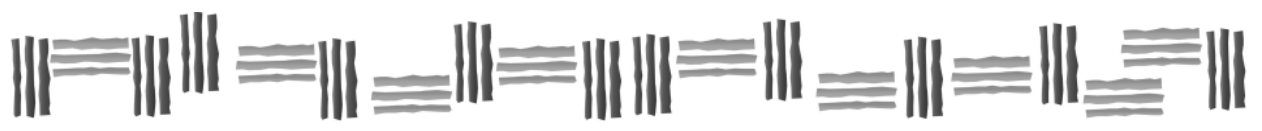




\section{Introducción.}

En este artículo se van a determinar los factores que más inciden en el potencial de desarrollo económico de un municipio, realizando una jerarquización de los mismos en base a dicho concepto. El primer problema que surge es el de la delimitación de la variable latente seleccionada y el método utilizado para medirla. El objetivo de este artículo no es otro que cuantificar el potencial de desarrollo económico de diferentes municipios, a partir de variables como la renta declarada, el nivel de paro registrado, la diversificación sectorial, etc. Todos ellos contienen aspectos positivos y, al mismo tiempo, presentan debilidades. Para resolver estos problemas los métodos se adaptan a los objetivos de los estudios planteados. Pero dicha medición nos permite, la que consideran Prieto y Delgado (2003: 98) como principal ventaja del modelo de Rasch, una "medición conjunta: los parámetros de ítems y personas están en la misma escala". Esto permite alcanzar un objetivo adicional: averiguar cuáles son los factores que más inciden en los resultados y con ello poder determinar en qué línea se deben concretar los progresos y la mejoría en la planificación de las políticas de desarrollo territorial de los municipios.

La medición del potencial económico no es una tarea fácil, ya que a veces los datos estadísticos no existen, son poco fiables, no contienen la información requerida, se reciben con mucho retraso, pierden vigencia rápidamente o no son buenos para mostrar el carácter multidimensional del potencial de desarrollo económico. En este artículo se va a elaborar una clasificación de los municipios seleccionados según su potencial de desarrollo económico, así como estudiar cuáles son los factores que más influyen en el grado de potencial que presentan cada uno de ellos. Se trata de determinar cómo los ítems seleccionados inciden en los niveles de potencial de desarrollo económico, de modo que permitan desvelar las claves de por qué un municipio ocupa esa posición y cómo la podría mejorar.

Para ello, en la primera parte de este artículo se realizará una revisión de la literatura sobre los intentos de mejorar la metodología en el diseño y planificación de las políticas de desarrollo territorial, objetivo del presente trabajo. En la segunda parte se aplicará el método de Rasch que permitirá desarrollar un análisis comparativo del potencial de desarrollo económico de dichos municipios, detallando su posición jerárquica, así como los factores determinantes que nos permitirán con más o menos intensidad mejorarla. Por último, se comentarán las conclusiones.

\section{Revisión de la literatura.}

Este apartado se va a comenzar con la enumeración de una serie de artículos que muestran la importancia desde el punto de vista investigador de cuestiones tales como las disparidades territoriales y el análisis de sus causas para conseguir atenuarlas; la importancia de las aportaciones de estudios empíricos como fundamento para orientar la planificación de las políticas públicas en aras de conseguir optimizar el potencial de desarrollo económico; determinar las capacidades y potencialidades propias de cada territorio desde un punto de vista estratégico (desarrollo endógeno) dentro de las nuevas políticas de abajo-arriba impulsadas por los actores locales, dada la incapacidad de las políticas macroeconómicas y sectoriales como instrumentos en un contexto de Unión Económica y Monetaria para salir de una situación de crisis económica como la que hemos experimentado recientemente; la diversidad económica y social del mundo rural y la importancia de su consideración a la hora de implementar políticas económicas; $\mathrm{y}$, por último, los problemas metodológicos que han aparecido en aspectos tales como de qué forma medir el modelo de crecimiento endógeno regional así como el nivel económico de los territorios, la necesidad de realizar un mayor uso de microdatos o datos por unidad y de integrarlos en estructuras espaciales más generales, etc.

Así, según Peña (2006), las disparidades económicas internas de una región influyen notablemente en las disparidades económicas externas (o entre regiones), limitando la capacidad de la misma para acercarse a los niveles de renta per cápita y empleo del resto de las regiones de su entorno, no siendo Andalucía una excepción tanto a nivel nacional como europeo. En el citado trabajo de investigación se pretende analizar la evolución y situación de las disparidades económicas intrarregionales en Andalucía y algunos de los factores explicativos de las mismas tanto a nivel provincial (modelo neoclásico) como bien a nivel comarcal (enfoque del potencial endógeno). Por tanto, 
la razón de este estudio es tratar de demostrar que las disparidades económicas intrarregionales en Andalucía están impidiendo un crecimiento sostenido y continuado que le permita converger con las regiones españolas y de la Unión Europea. En la misma línea, Peña (2010) pretende poner de relieve cuál ha sido la evolución de las disparidades económicas territoriales en el interior de la provincia de Cádiz en el periodo 1991-2000, partiendo del hecho de que los distintos municipios han experimentado tasas de crecimiento desiguales en relación con el Valor Añadido Bruto y la población, que se han traducido en un comportamiento también diferenciado de las desigualdades en VAB por habitante. Por otro lado, analiza algunos factores explicativos de las disparidades económicas municipales en la provincia de Cádiz, tales como la aglomeración de población, la productividad y la estructura sectorial.

García (2014) muestra cómo los resultados de estudios empíricos se pueden emplear con el fin de proporcionar orientación valiosa al diseño y gestión de las políticas públicas; éstas son las ventajas derivadas de la cooperación entre quienes se encargan de la formulación de las políticas, quiénes las ponen en práctica y quiénes se dedican a la investigación, a fin de maximizar el impacto y alcance de las políticas públicas en general. Todos los agentes implicados en la toma de decisiones en materia de políticas públicas, están de acuerdo sobre la creciente importancia atribuida a políticas y programas que están respaldadas por resultados basados en la evidencia empírica. En inglés, este término se denomina evidence-based policy. Un enfoque y gestión de políticas basadas en la evidencia es aquél que se refiere a un proceso político que ayuda a los planificadores a tomar decisiones bien documentadas sobre políticas, programas y proyectos, al proveerse de la mejor y más precisa evidencia, y hacer de ésta el punto central del desarrollo e implementación de las políticas o del proceso político.

Entrena y Álvarez (2014) llevan a cabo una investigación sobre las dinámicas sociodemográficas y económicas en la Comarca española de Guadix, haciendo hincapié en la necesidad de incidir en políticas de desarrollo rural más adecuadas y efectivas para esta zona rural en declive. Ello es porque a pesar de la continua intervención en desarrollo, las pérdidas demográficas siguen siendo significativas; sobre todo, de los efectivos poblacionales más jóvenes. Sin embargo, debido a su localización geográfica y a la calidad de sus cuantiosos recursos socioculturales y naturales, se trata de un territorio sostenible social y ambientalmente, en el que además tales recursos constituyen factores potenciales de desarrollo socioeconómico.

El trabajo de De Haro et al. (2017) realiza una evaluación tanto del desarrollo económico como de su potencial a nivel municipal; concretamente identifica desigualdades entre los municipios del estado de Nayarit, México, a partir de un análisis de divergencias con los índices de desarrollo socioeconómico y potencial de desarrollo socioeconómico. Los indicadores que componen el nivel de desarrollo socioeconómico son el índice de marginación, el grado de urbanización, la tasa bruta de actividad económica, el coeficiente de dependencia económica y la densidad de carreteras pavimentadas. Por otra parte, los indicadores que componen el nivel de potencial de desarrollo socioeconómico son la situación geográfica de los municipios, la densidad de población, el grado de cualificación de la población, la concentración sectorial de las funciones secundarias y terciarias y el coeficiente de suficiencia de la red vial.

A partir de ellos, puede observarse que la distribución de los niveles tanto de desarrollo como de potencial de desarrollo es regular, concentrándose los rangos menores en la zona serrana del estado y las condiciones de mayor ventaja en la zona centro, donde se localiza la capital. Los resultados muestran diferencias significativas. Los factores que presionan hacia una mayor desigualdad socioeconómica son: marginación, escasa actividad económica, deficiente estructura carretera, bajo nivel de cualificación de la población y baja ocupación en actividades económicas secundarias y terciarias. De los veinte municipios analizados, Del Nayar, La Yesca y Huajicori presentan la mayor desventaja, mientras que Tepic muestra la mejor condición.

Granados et al. (2016) tratan de identificar las capacidades y potencialidades propias con las que cuenta el Área Metropolitana del Valle de Aburrá, para el desarrollo territorial. El proceso de investigación está compuesto por dos momentos metodológicos: el primero es la identificación de capacidades, restricciones y potencialidades con las que cuenta el territorio, y el segundo es un análisis de la percepción de los expertos o agentes de desarrollo que intervienen en la economía del territorio, para así identificar posibles estrategias que promuevan la competitividad. 
Según Reig (2010) los municipios rurales presentan una considerable heterogeneidad en relación a su potencial de desarrollo, por lo que la correcta caracterización del nivel socioeconómico de cada uno de ellos es un requisito básico en la elaboración de políticas de desarrollo rural. El reconocimiento de la diversidad socioeconómica del mundo rural es una condición indispensable para diseñar políticas apropiadas de desarrollo rural que atiendan a las distintas condiciones imperantes en diferentes zonas o regiones.

Nunes y Karnopp (2015) analizan las potencialidades de desarrollo endógeno de Julio de Castilhos, localizado en la Región Central del Estado de Río Grande del Sur, con menos de 50 mil habitantes y con base económica en el sector primario. El problema que se investiga es delimitar cuáles son las potencialidades endógenas de desarrollo de Julio de Castilhos - RS, utilizándose metodológicamente un enfoque deductivo, defendiéndose un posicionamiento estratégico para aprovechar las oportunidades locales y regionales.

El artículo de Ruiz y Becerra (2015) propone un sistema de indicadores para generalizar el análisis, la evaluación y el impacto de los proyectos de desarrollo local. El modelo es aplicable tanto a proyectos de la Iniciativa Municipal de Desarrollo Local como a los que reciben la financiación por la vía de la colaboración extranjera y muestra su aplicación en el municipio Cienfuegos. Esta investigación permite evaluar su eficacia, eficiencia, pertinencia, impacto -económico, social y ambiental- y sostenibilidad. Se parte de un axioma referido por varios autores consistente en que "no se puede mejorar lo que no se puede medir", y de ahí, surge la propuesta de un sistema de indicadores que permita evaluar, integralmente, estos proyectos de desarrollo y que incluya, además, su impacto económico, social y ambiental.

Gaviria (2013) refleja cómo frente a la incapacidad mostrada por los gobiernos centrales para resolver, a través de políticas macroeconómicas y sectoriales, los problemas de los territorios a nivel regional y local, fueron apareciendo primero en Europa en el decenio de los ochenta y luego en América latina en los años noventa, nuevas políticas de abajo-arriba impulsadas por los actores locales. Así, en dicho trabajo se estudia la situación del municipio de Santa Rosa de Cabal, a partir de la revisión de las potencialidades productivas, organizativas e institucionales desde las que se puede dinamizar un proceso de desarrollo local endógeno.

Según Caravaca et al. (2014), la inserción de empresas y territorios en un mundo globalizado está condicionada, entre otros factores, por su capacidad para incorporar conocimiento y realizar innovaciones. No es de extrañar que dichas capacidades se conviertan en el punto de confluencia de diferentes corrientes epistemológicas y disciplinas científicas que reflexionan acerca de si pueden constituir la base de un nuevo modelo socioeconómico. Junto a lo anterior, emerge una nueva cultura territorial que dedica una atención especial a las ciudades medias, al considerar que pueden inducir efectos multiplicadores sobre su entorno y contrarrestar así los procesos concentradores provocados por las grandes ciudades.

En este artículo se pretende realizar un análisis comparado de algunas ciudades medias en una región del sur de España, Andalucía, desde varias perspectivas: su grado de inserción en la sociedad del conocimiento, su capacidad innovadora y las redes locales de colaboración que pueden propiciarla, y las estrategias públicas de desarrollo local que en ellas se están implementando.

En este sentido, para Santinha (2014) la valorización de la dimensión territorial en la formulación de las políticas públicas ha sido visible en las orientaciones emanadas por las más diversas instituciones mundiales, lanzando recientemente para el debate político y académico la Cohesión Territorial en cuanto nuevo paradigma de desarrollo. En un momento de particular importancia, en que se discuten las orientaciones para el periodo pos-2013 y la atribución de los fondos estructurales, esta cuestión asume un interés adicional en los estados miembros de la Unión Europea. A pesar de que, como objetivo político, la cohesión territorial ha alcanzado una importancia elevada, todavía es notoria la ausencia de una delimitación precisa del concepto, dificultando la capacidad de lidiar de forma analítica y normativa con este principio. Es sobre esta cuestión que el texto se concentra, identificándose caminos que podrán contribuir como referencial para la formulación y territorialización de políticas públicas, mejorando, así, los procesos de decisión con base en este principio. 
Según Varela (2015), se analiza la emergencia de un nuevo paradigma que determina roles diferenciados y contrapuestos a los esquemas clásicos vigentes en los gobiernos locales, particularmente en las regiones metropolitanas, desarrollados como resultado de tendencias globales. Tales cambios, provenientes de la economía, la mercantilización de los bienes públicos y la liberalización e integración de los mercados, afectan los diseños institucionales tradicionales, que les otorgan competencias delimitadas y subordinadas a los gobiernos locales en el marco de un modelo soberanista centrado en el estado-nación. Con la globalización, este esquema ha cambiado y hoy los gobiernos locales asumen en las grandes ciudades nuevas competencias, que corresponden a la emergencia de nodos metropolitanos que se articulan con los mercados globales y con dinámicas de reconfiguración de flujos económicos, políticos, sociales y demográficos. Este proceso ocurre en términos de redes de ciudades y de integración de regiones con esquemas asimétricos de concentración de recursos y poder, modificando el mapa del desarrollo territorial. Por tanto, aparecen nuevos roles a desarrollar por los gobiernos locales en la implementación de políticas públicas.

Dado que el paradigma del "desarrollo local" está hoy plenamente consolidado, Esparcia et al. (2016) analizan y conceptualizan los procesos de desarrollo local prestando atención también a la dimensión social y no solo a la económica, y, en particular, a la componente relacional del capital social.

Según Stimson (2016), los científicos regionalistas se han enfrentado a numerosos desafíos en el desarrollo del ámbito interdisciplinario en el que analizan el contexto espacial de los fenómenos sociales, económicos y medioambientales, y asimismo tratar con amplias bases de datos plantea desafíos metodológicos considerables. Este artículo estudia la evolución de la Ciencia Regional, las críticas que ha recibido y los retos con los que se ha enfrentado. Analiza específicamente, entre otros, algunos retos contemporáneos relacionados con problemas metodológicos como de qué forma medir y los logros del modelo de crecimiento endógeno regional; la necesidad de realizar un mayor uso de microdatos o datos por unidad y de integrarlos en estructuras espaciales más generales...

Esta breve revisión bibliográfica trata de poner de manifiesto la candencia de los citados aspectos, que inspiran el presente trabajo, que, a pesar de ello, muestra unas señas de identidad propias.

En segundo lugar, en el presente apartado queremos señalar que Gutiérrez (2007: 31-32) plantea su modelo como una alternativa para valorar el potencial de desarrollo económico de los territorios y el grado de vulnerabilidad de los mismos. De tal modo, que una parte de dicha evaluación la lleva a cabo tomando como base el enfoque del modelo de Biehl (1988), que considera solo la incidencia sobre el constructo teórico mencionado de los cuatro factores de potencialidad siguientes: la localización, la aglomeración, la estructura sectorial y la infraestructura. Sin embargo, por otra parte, complementa la evaluación anterior, de tal manera que en la definición del potencial de desarrollo económico territorial llevada a cabo en el modelo ampliado y revisado de Gutiérrez, también se consideran los denominados factores limitantes del desarrollo regional, incluyendo en este sentido la pobreza, la desigualdad y la marginación, radicando el sustento teórico de dicho modelo en la relación existente entre ambos tipos de factores, derivándose precisamente de dicha relación la información valiosa y diferente a la del modelo de Biehl, erigiéndose el modelo ampliado de Gutiérrez, por tanto, como un avance en los instrumentos que tratan de evaluar la capacidad de los territorios, no exclusivamente para crecer y desarrollarse, sino también para hacer frente de manera adecuada a los cambios económicos (este hecho se trata de analizar en el presente estudio mediante la realización de una comparación entre dos momentos temporales totalmente distintos, desde el punto de vista del ciclo económico, en las dos mediciones efectuadas de los municipios). Este autor concluye que su modelo teniendo en cuenta los resultados empíricos obtenidos en su estudio es una herramienta útil para el análisis del potencial de desarrollo económico de los territorios, así como para contribuir a una planificación más eficiente de la política económica destinada a estimular su crecimiento y desarrollo, reducir sus puntos débiles y lograr la convergencia en términos de empleo, renta y riqueza, aunque previamente a cualquier propuesta de política territorial señala que es preciso analizar el contexto y las características peculiares de la unidad territorial en cuestión, puesto que no hay una receta universal para lograr dicho proceso de desarrollo.

El presente trabajo de investigación se puede insertar, sin lugar a dudas, en el marco genérico de discusión expuesto en las líneas anteriores, puesto que partiendo de la consideración de las líneas maestras marcadas por dicho cuerpo teórico se han realizado una serie de mediciones mediante la 
aplicación del modelo de Rasch. Sin embargo, no existe la posibilidad de comparar los resultados concretos obtenidos con los de otros análisis empíricos de la misma índole debido a que no se han encontrado estudios previos llevados a cabo mediante la aplicación de la citada metodología a unos escenarios definidos con los mismos criterios, con unos ítems similares y con un nivel de desagregación municipal de los datos.

Los factores de potencialidad citados han servido de inspiración para delimitar los ítems seleccionados.

La aglomeración o concentración espacial de población y/o actividades económicas, reseñada como uno de los factores de potencialidad del desarrollo económico de cualquier región o zona tanto en el modelo de Biehl como en el de Gutiérrez, no se incluye directamente en el presente análisis sino de manera indirecta, puesto que todos los ítems se han relativizado en función de sus características bien respecto a la población total (ingresos presupuestarios por habitante) bien respecto a la población económicamente activa (el resto de los ítems, salvo el índice de concentración sectorial que se expresa en términos absolutos, se presentan en relación a la población de 16 a 64 años, puesto que se considera que es la realmente relevante de cara al desarrollo potencial, además de que los ítems 1-4 al tratarse de variables de naturaleza estrictamente económica están correlacionados positivamente, mientras que el ítem 5 lo estará negativamente, con un incremento de la concentración espacial de la actividad económica, mostrando como consecuencia un mayor o menor valor cuanto mayor sea la concentración de dichas actividades en una zona; quedando, por tanto, el citado factor incluido en el estudio de forma implícita o indirecta).

En relación al concepto de infraestructuras de Biehl, se ha de decir que la capacidad de dicho factor a la hora de influir en el potencial de desarrollo económico de un municipio estaría relacionada con el nivel de los ingresos presupuestarios por habitante, puesto que un crecimiento en este ítem posibilitaría a los poderes públicos proporcionar un mayor nivel de infraestructuralidad a dicho ámbito territorial (se considera que se encuentra por debajo de su nivel potencial) y, por tanto, según los modelos teóricos considerados, generar un mayor potencial de desarrollo económico municipal. El logro de una mayor dotación de infraestructuras se traduciría a su vez en unos mayores niveles de ingreso, productividad y empleo del territorio $\mathrm{y}$, como consecuencia, en unos ingresos presupuestarios nuevamente más elevados, originándose de esta forma una retroalimentación o feed-back que se traduciría en un círculo virtuoso positivo en términos de potencial de desarrollo económico. Además, este mecanismo reduciría los niveles de pobreza, marginación y desigualdad, obstaculizando de esta manera el efecto negativo de los factores limitantes del potencial de desarrollo económico.

Como se puede observar, el ítem 6 refleja la composición sectorial de la estructura productiva que tiene cualquier municipio, tratándose, por tanto, de uno de los 4 factores de potencial de desarrollo económico de cualquier región o zona considerado tanto en el modelo de Biehl como en el de Gutiérrez. Siguiendo el análisis ampliado del modelo de Biehl llevado a cabo por Gutiérrez (2007), se considera de manera implícita la inclusión de los factores limitantes del desarrollo regional: pobreza, marginación, y desigualdad, de tal manera que los otros cinco ítems contienen variables estrictamente económicas que de alguna manera englobarían dichos factores restrictivos. Es decir, siguiendo la línea de pensamiento de Gutiérrez se considera que aquellos municipios que presentan valores elevados de los ítems 1, 2, 3 y 4, y valores reducidos de paro por habitante en edad de trabajar, presentaran menores niveles de pobreza, marginación y desigualdad, y como consecuencia, un mayor nivel de potencial de desarrollo económico.

Por último, destacar que la metodología elegida, el Modelo de Rasch, es una técnica muy actual y apropiada de cara a su aplicación a investigaciones en ciencias sociales como la que se presenta en este trabajo. Un máximo exponente de dicha afirmación puede encontrarse, por ejemplo, en el trabajo de Cuenca et al. (2019), que además guarda muchas similitudes con el que se expone en estas líneas puesto que también analiza el nivel económico de los territorios, concretamente analiza los factores determinantes de las diferencias en el grado de pobreza entre países a través del Modelo de Rasch. 


\section{Datos y metodología: el modelo de Rasch.}

La presente investigación utiliza un método inductivo mediante un proceso analítico-sintético que pretende cuantificar la variable latente "Potencial de desarrollo económico de un municipio". Así, se parte de la descomposición de dicho objeto de estudio en una serie de ítems, que se estudian de forma individual y comparativa en dos momentos temporales distintos (análisis) y luego se procede a sintetizar dichas partes en una medida única para estudiarlas de manera holística e integral. Todo este proceso se ha llevado a cabo aplicando el método métrico de Rasch.

El atributo estadístico que diferencia al modelo de Rasch es que los parámetros personas e ítems están algebraicamente separados y dan lugar a estadísticos suficientes (Andersen, 1973; Masters \& Wright, 1984). Rasch considera que, si los ítems elegidos son los correctos, surgirán pocos errores, por lo que es razonable representar la distribución de los errores de lectura mediante una función de probabilidad de Poisson (multiplicativa). La elección de este modelo de Poisson multiplicativo se debía a las propiedades que le cualifican como un modelo de medida (Lord, 1953). El estudio de los atributos que debían verificar las medidas, llevan a Rasch a la utilización de modelos aditivos exponenciales (modelos de medida): pasando del modelo de Poisson multiplicativo al modelo logístico. Rasch desarrolló su modelo dentro de la teoría de la medición conjunta, que parte de la consideración de que las medidas (observables) fundamentales tienen estructura aditiva. Perline et al. (1979) han probado que los modelos que están incluidos en la familia identificada por Rasch son los únicos modelos compatibles con los principios de la teoría de la medida conjunta en el caso probabilístico. Este hecho otorga un papel crucial a los modelos de medida de Rasch en la metodología para la medición en las Ciencias Sociales (Cliff, 1992; Michell, 1999). Así, los modelos de Rasch han sido aplicados en psicología, pedagogía, economía aplicada, salud pública, etc., para la confección de instrumentos de medida o identificación de continuos de medida en un conjunto de datos ya disponibles.

Siguiendo a Alvarado y Santisteban (2006: 10), el instrumento de medida propuesto por Rasch supone tanto la posibilidad de obtener estimadores suficientes de los parámetros, con medidas directas, y haciendo uso de las propiedades de las distribuciones y de los procesos de Poisson compuestos, como la de verificar los requisitos teóricamente exigibles: la estabilidad, la separabilidad, la independencia muestral, así como el requisito imprescindible de aditividad conjunta. Se convierte así en una herramienta de medida de variables latentes que, además, posibilita valorar el ajuste de las respuestas a los ítems y el que se produce entre la respuesta de un sujeto y los patrones de respuesta de la muestra a la que éste pertenece (Febles, 2008: 17).

Esta técnica presenta planteamientos sencillos, dado que la respuesta a un ítem depende tanto de la preparación y competencia del sujeto como de la dificultad del ítem (Febles, 2008: 17). Así, según Sánchez y Blanco (2012: 37-38), ofrece las siguientes ventajas: es capaz de extraer conclusiones fiables a partir de datos particulares o muestras pequeñas (suficiencia estadística); identifica casos que presentan desajustes respecto al conjunto de los datos, lo que lo diferencia de otras técnicas donde los posibles desajustes son eliminados del análisis por considerar que desvirtúan la muestra (Rasch los identifica para tratar de encontrar una justificación a dicha anomalía, puesto que suele ser muy clarificador en el caso de hechos o fenómenos económicos); estudia la fiabilidad de los sujetos y de los ítems de forma individual; calcula la fiabilidad y la validez global de las medidas; comprueba la hipótesis de unidimensionalidad; analiza las categorías de las respuestas, jerarquizando los ítems en función de su dificultad y los sujetos en función de su habilidad; identifica distintos grupos de sujetos enfrentando las mediciones con otras variables diferenciales como el tamaño, la identidad rural o metropolitana, la distancia a la capital... e identifica los puntos fuertes y débiles de cada sujeto de forma individual.

En definitiva, la Quantum Measurement Technique, basada en la Probabilidad de Rasch, es un instrumento de trabajo que permite obtener la medida de una variable latente, en este caso concreto "Potencial de desarrollo económico de un municipio", siendo una medida más adecuada que otras al reducir matrices complejas de datos a una variable unidimensional (Morán \& Álvarez, 2001: 196). Los parámetros que rigen la probabilidad de Rasch se han hallado utilizando el programa informático Winsteps Rasch Measurement (Linacre, 2014). 
Así, en el presente trabajo se ha aplicado dicha Probabilidad a todos los municipios de la provincia de Sevilla, obteniéndose inicialmente 105 municipios, de los que deben suprimirse 7 (Albaida del Aljarafe, Burguillos, Huévar del Aljarafe, Pedrera, Tocina, Villanueva de San Juan, Villanueva del Ariscal) debido a que para estos municipios no se dispone de datos en SIMA del año 2007 para el ítem ingresos presupuestarios por habitante. Por tanto, finalmente quedan para la medida 98 municipios, recogidos en la Tabla 1. Para la comparación intertemporal se eligen tanto el año 2007 (último año de crecimiento económico antes de la crisis) como el año 2012 (último año de recesión económica para el cual disponemos de datos completos). Todo ello ha permitido cuantificar los ítems para los distintos municipios y conocer con detalle el potencial de desarrollo económico de los mismos, caracterizados por su pertenencia a la provincia de Sevilla.

Los parámetros que rigen la probabilidad de Rasch se han hallado utilizando el programa informático Winsteps, concretamente su versión 3.81.0. El resultado final es una medida del potencial de desarrollo económico para cada municipio y para cada ítem, así como los desajustes tanto para unos como para otros.

Dada la variable latente "x", medida para los 98 municipios tanto para el año 2007 como para el año 2012, y definida por un conjunto de 6 ítems no correlacionados, esta técnica de medición los sitúa a lo largo de una línea para su medida conforme a su situación de potencial de desarrollo económico (Morán \& Álvarez, 2001: 120), evaluando éstos conforme a la escala de valores del 1 (valor inferior) al 10 (valor superior). Los atributos que se han utilizado para analizar este constructo quedan reflejados en 6 ítems cuya numeración coincide además con el número de registro o entrada en el programa informático que se ha utilizado para realizar las mediciones (Winsteps):

- Ítem no 1: Número de declaraciones/Población en edad de trabajar (de 16 a 64 años).

- Ítem no 2: Rentas netas del trabajo/Población en edad de trabajar (de 16 a 64 años).

- Ítem $n^{\circ}$ 3: Ingresos presupuestarios por habitante.

- Ítem no 4: Contratos registrados/Población en edad de trabajar (de 16 a 64 años).

- Ítem no 5: Paro registrado/Población en edad de trabajar (de 16 a 64 años), que requiere de un tratamiento sobre su impacto inverso.

- Ítem $\mathrm{n}^{\mathrm{o}}$ 6: Índice de concentración sectorial de la estructura productiva (un valor bajo de este índice indicaría una elevada diversificación sectorial de la estructura productiva, de ahí que tenga un tratamiento inverso).

El ítem Índice de concentración sectorial se ha calculado a partir del Índice de Herfindahl. Este cálculo se expresa de la siguiente fórmula $H=\sum_{i=1}^{N} S_{i}^{2}$, que es la suma de los cuadrados de los tamaños relativos de las empresas de la industria considerada. En su aplicación a este caso concreto, se ha considerado por analogía como la suma de los cuadrados de los tamaños relativos de los sectores del municipio considerado, es decir, este índice se basa en el número total de sectores y en la distribución de los tamaños de los sectores productivos de un municipio. El Índice de Herfindahl se puede calcular sobre una base 1, donde un $\mathrm{HHI}=1$ indica que en la estructura productiva del municipio solo hay un sector, sobre una base de $1.000 \mathrm{o} 10.000$. Por tanto, si "x" es la variable latente definida por un conjunto de ítems, entonces estos ítems son las características consideradas como más relevantes para explicar el potencial de desarrollo económico municipal, teniendo en cuenta la disponibilidad de información y que no presentan grados de correlación significativos. 
Tabla 1. Municipios de la provincia de Sevilla.

\begin{tabular}{|c|c|c|c|c|c|c|c|c|c|c|c|c|c|}
\hline 1 & Aguadulce & 15 & $\begin{array}{l}\text { Bollullos de la } \\
\text { Mitación }\end{array}$ & 29 & $\begin{array}{l}\text { Castilleja del } \\
\text { Campo }\end{array}$ & 43 & El Garrobo & 57 & El Madroño & 71 & Paradas & 85 & $\begin{array}{l}\text { San Juan de } \\
\text { Aznalfarache }\end{array}$ \\
\hline 2 & Alanís & 16 & Bormujos & 30 & $\begin{array}{l}\text { El Castillo de las } \\
\text { Guardas }\end{array}$ & 44 & Gelves & 58 & $\begin{array}{l}\text { Mairena del } \\
\text { Alcor }\end{array}$ & 72 & El Pedroso & 86 & $\begin{array}{l}\text { San Nicolás del } \\
\text { Puerto }\end{array}$ \\
\hline 3 & $\begin{array}{l}\text { Alcalá de } \\
\text { Guadaíra }\end{array}$ & 17 & Brenes & 31 & Cazalla de la Sierra & 45 & Gerena & 59 & $\begin{array}{l}\text { Mairena del } \\
\text { Aljarafe }\end{array}$ & 73 & Peñaflor & 87 & Sanlúcar la Mayor \\
\hline 4 & \begin{tabular}{|l|} 
Alcalá del \\
Río
\end{tabular} & 18 & $\begin{array}{l}\text { Las Cabezas de } \\
\text { San Juan } \\
\end{array}$ & 32 & Constantina & 46 & Gilena & 60 & Marchena & 74 & Pilas & 88 & Santiponce \\
\hline 5 & $\begin{array}{l}\text { Alcolea del } \\
\text { Río }\end{array}$ & 19 & Camas & 33 & Coria del Río & 47 & Gines & 61 & Marinaleda & 75 & Pruna & 89 & El Saucejo \\
\hline 6 & La Algaba & 20 & La Campana & 34 & Coripe & 48 & Guadalcanal & 62 & $\begin{array}{l}\text { Martín de la } \\
\text { Jara }\end{array}$ & 76 & $\begin{array}{l}\text { La Puebla de } \\
\text { Cazalla }\end{array}$ & 90 & Sevilla (capital) \\
\hline 7 & Algámitas & 21 & Cantillana & 35 & El Coronil & 49 & Guillena & 63 & Los Molares & 77 & $\begin{array}{l}\text { La Puebla de los } \\
\text { Infantes }\end{array}$ & 91 & Tomares \\
\hline 8 & $\begin{array}{l}\text { Almadén de } \\
\text { la Plata }\end{array}$ & 22 & Cañada Rosal & 36 & Los Corrales & 50 & Herrera & 64 & Montellano & 78 & La Puebla del Río & 92 & Umbrete \\
\hline 9 & Almensilla & 23 & Carmona & 37 & $\begin{array}{l}\text { El Cuervo de } \\
\text { Sevilla }\end{array}$ & 51 & Isla Mayor & 65 & $\begin{array}{l}\text { Morón de la } \\
\text { Frontera }\end{array}$ & 79 & El Real de la Jara & 93 & Utrera \\
\hline 10 & Arahal & 24 & $\begin{array}{l}\text { Carrión de los } \\
\text { Céspedes }\end{array}$ & 38 & Dos Hermanas & 52 & La Lantejuela & 66 & $\begin{array}{l}\text { Las Navas de la } \\
\text { Concepción }\end{array}$ & 80 & La Rinconada & 94 & $\begin{array}{l}\text { Valencina de la } \\
\text { Concepción }\end{array}$ \\
\hline 11 & Aznalcázar & 25 & Casariche & 39 & Écija & 53 & Lebrija & 67 & Olivares & 81 & $\begin{array}{l}\text { La Roda de } \\
\text { Andalucía }\end{array}$ & 95 & $\begin{array}{l}\text { Villamanrique de la } \\
\text { Condesa }\end{array}$ \\
\hline 12 & Aznalcóllar & 26 & $\begin{array}{l}\text { Castilblanco de los } \\
\text { Arroyos }\end{array}$ & 40 & Espartinas & 54 & Lora de Estepa & 68 & Osuna & 82 & El Ronquillo & 96 & $\begin{array}{l}\text { Villanueva del Río y } \\
\text { Minas }\end{array}$ \\
\hline 13 & Badolatosa & 27 & $\begin{array}{l}\text { Castilleja de } \\
\text { Guzmán } \\
\end{array}$ & 41 & Estepa & 55 & Lora del Río & 69 & $\begin{array}{l}\text { Palacios y } \\
\text { Villafranca } \\
\end{array}$ & 83 & El Rubio & 97 & Villaverde del Río \\
\hline 14 & Benacazón & 28 & $\begin{array}{l}\text { Castilleja de la } \\
\text { Cuesta }\end{array}$ & 42 & $\begin{array}{l}\text { Fuentes de } \\
\text { Andalucía } \\
\end{array}$ & 56 & La Luisiana & 70 & \begin{tabular}{|l|} 
Palomares del \\
Río
\end{tabular} & 84 & Salteras & 98 & El Viso del Alcor \\
\hline \multicolumn{14}{|c|}{ 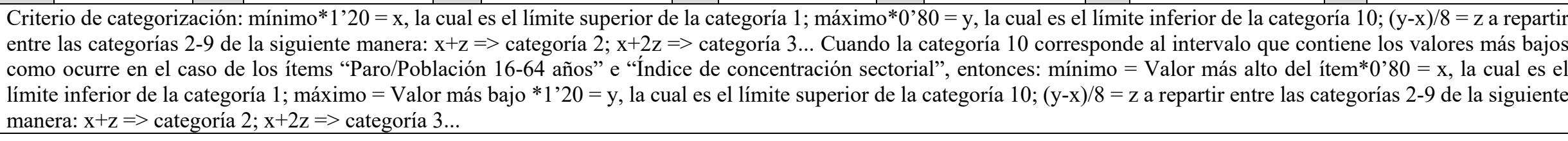 } \\
\hline
\end{tabular}

Fuente: SIMA (Instituto de Estadística y Cartografía de Andalucía). 
Como cualquier otra variable latente, el potencial de desarrollo económico puede visualizarse como una línea con una dirección a lo largo de la que se sitúan los ítems y los municipios. Esta representación muestra que el municipio $\beta_{0}$ no sobrepasa ningún ítem por lo que estará entre los municipios con mayores debilidades. El municipio $\beta_{1}$ sobrepasa sólo al ítem $\delta_{1}$, el municipio $\beta_{2}$ sobrepasa los ítems $\delta_{1}$ y $\delta_{2}$, mientras que el municipio $\beta_{3}$ sobrepasa los tres ítems y será el de mayor potencial de desarrollo económico. El ítem $\delta_{1}$ es el de menor medida, lo que representa una fortaleza más fácil de alcanzar $y$, como consecuencia, un nivel de potencial de desarrollo económico más habitual de conseguir, logrado por los municipios $\beta_{1}, \beta_{2}$ y $\beta_{3}$; mientras que el ítem $\delta_{3}$ es el de mayor medida (el que está más a la derecha) y representa un nivel de potencial de desarrollo económico más raro, siendo sólo alcanzado por el municipio $\beta_{3}$.

Figura 1. Continuo Lineal.

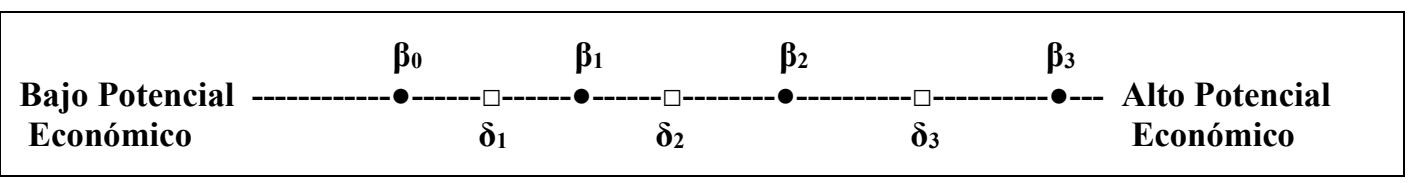

Fuente: Elaboración propia a partir de Oreja (2015:53).

Si se considera $X_{n i}$ como la variable dicotómica potencial de desarrollo económico municipal que describe el hecho de que un municipio " $n$ " endosa el ítem " $i$ ". Si $X_{n i}=1$, entonces el municipio " $n$ " se dice que es potente; por el contrario, si $\mathrm{X}_{\mathrm{ni}}=0$ se dice que el municipio " $\mathrm{n}$ " no es potente. Rasch empleó una función logística para explicar la relación entre la probabilidad de la opción correcta y la probabilidad de una opción incorrecta (ver Prieto \& Delgado, 2003: 94-95).

Con los cálculos apropiados se obtiene de la fórmula que se presenta, mostrando en el presente caso la probabilidad de que el municipio " $n$ " referido al ítem "i" sea potente, dados los parámetros $\beta_{\mathrm{n}} \mathrm{y}$ $\delta_{\text {i. }}$ Ésta es la fórmula que George Rasch obtuvo en su tratado acerca de las variables latentes (Morán \& Álvarez, 2001: 198).

$$
P\left[X_{n i}=1\right] \beta_{n}, \delta_{i}=\frac{e^{(\beta n-\delta i)}}{1+e^{(\beta n-\delta i)}}
$$

Una herramienta de medida configurada con estos ítems, posibilita el poder medir el potencial de desarrollo económico de un municipio y, además, permite posicionar a cada municipio estudiado dentro del ámbito de estudio elegido.

El presente estudio se centrará en el análisis del potencial de desarrollo económico de los municipios considerados, atendiendo a criterios de renta, empleo y diversificación productiva.

Partiendo de que lo que se quiere analizar es el nivel de potencial de desarrollo económico de los municipios elegidos, el objetivo fundamental de este trabajo se puede dividir en dos:

- Posicionar a cada municipio dentro del conjunto elegido para su comparación.

- Determinar qué factores condicionan dicho posicionamiento.

Se ha de señalar que el modelo de Rasch es politómico (Rasch, 1980; Andrich, 1978, 1988a y 1988b), de tal modo que en el caso que se está considerando la puntuación asignada se representa por la escala de categorías $\{1,2,3,4,5,6,7,8,9,10\}$ en términos del parámetro sujeto (municipios) e ítem (factores de potencial de desarrollo económico de un municipio).

Por tanto, debido a que en el presente trabajo los datos originales de los municipios no aparecen categorizados y toman valores de acuerdo a diferentes escalas y porcentajes, es preciso, conforme al modelo de Rasch de categorías ordenadas de Andrich-Rasch (Andrich, 1978), categorizar de forma conjunta a una misma escala todos los ítems del instrumento de medición diseñado. 
El objetivo de esta categorización es transformar varias medidas que representan conceptos de diferente naturaleza o índole, en una medida global que simplifica y permite una interpretación conjunta (Álvarez, 2008).

De este modo, una vez obtenidos los datos de SIMA, el siguiente paso fue hallar los valores máximo y mínimo de cada factor de potencialidad o ítem, y establecer un criterio de valoración que convirtiera los datos extraídos en datos recogidos en un intervalo de 1 a 10 , con el objetivo de estandarizarlos para poder tratarlos a través del modelo de Rasch (1980), con el programa Winsteps (Linacre, 2014).

Así, para llevar a cabo la categorización de los indicadores o ítems se utilizan 10 categorías, calculándose 10 intervalos o tramos para cada ítem, asignándose a cada uno de ellos los valores 1,2 , $3 . . .10$, según se recoge en nota en la Tabla 1. En este sentido, se ha de resaltar también que dos de los ítems utilizados, concretamente Paro/Población en edad de trabajar e Índice de Concentración Sectorial, presentan una polaridad invertida, es decir, que un mayor valor del indicador, muestra un peor nivel de potencial de desarrollo económico.

La finalidad de este modelo es simplemente ubicar tanto los municipios seleccionados como los factores de potencial de desarrollo económico municipal en una escala lineal unidimensional representativa del constructo teórico que posibilite contrastar resultados entre los municipios analizados y la relevancia de los ítems. Así, gracias al modelo se puede realizar un estudio detallado de los municipios y de las variables elegidas ya que estos se ubican en un mismo continuo lineal de forma simultánea, apareciendo, por tanto, en dicha línea de manera conjunta los municipios y los factores de potencial de desarrollo económico municipal seleccionados, determinándose una sola dirección en la que se considera que el extremo superior e inferior de la línea representan "alto" y "bajo", respectivamente, de la variable en cuestión, consiguiéndose así una jerarquía que llevará a la relación que se deriva de los municipios y su nivel de potencial de desarrollo económico, así como a la de la relevancia de los factores determinantes del mismo.

\section{Resultados empíricos.}

El posicionamiento de los 98 municipios en relación con su nivel de potencial de desarrollo económico se puede ver gráficamente a través del continuo lineal o mapa de Wright (Linacre, 2014) representado en la Tabla 2 tanto para 2007 como para 2012, respectivamente, en la que como ya se ha comentado se integran las medidas de los municipios y de los factores de potencial de desarrollo económico.

El continuo lineal se mueve en este caso entre +1 y -1 y se puede apreciar que está segmentado en torno de la media (M), la desviación típica (S) y dos veces la desviación típica (T). Partiendo de dicho mapa, se puede destacar que en el año 2007 el municipio con mayor potencial de desarrollo económico es Espartinas, mientras que el municipio con menor potencial es Castilblanco de los Arroyos. Del mismo modo, para el año 2012, el municipio con mayor potencial de desarrollo económico es nuevamente Espartinas, mientras que, para ese mismo año, el municipio con menor potencial es Villanueva del Río y Minas. 
Tabla 2. Mapa de sujetos e ítems para los municipios de la provincia de Sevilla.

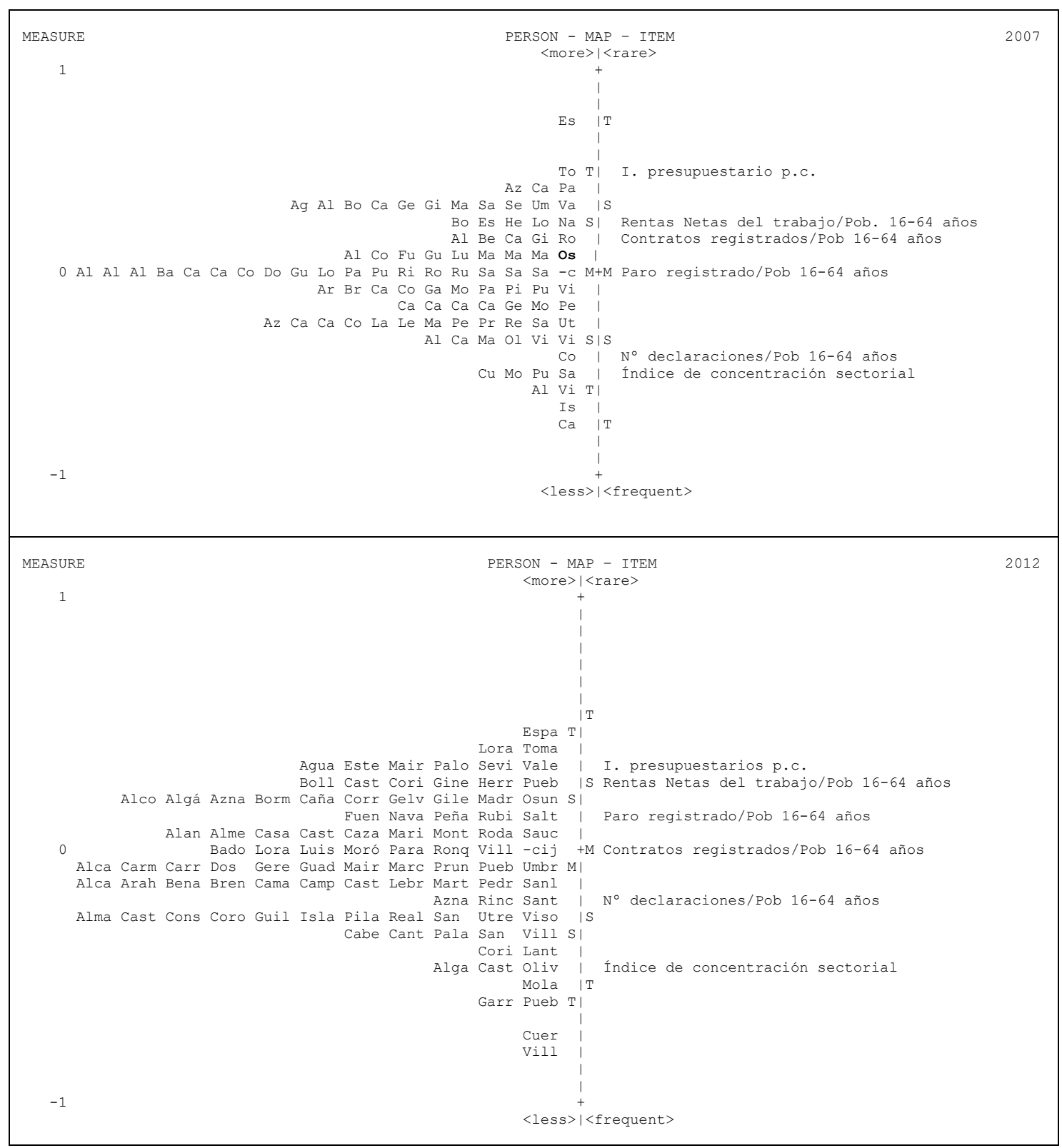

Fuente: Elaboración propia a partir de Winsteps 3.81.0.

Asimismo, en dicho mapa se puede apreciar que, en ambos años, el factor de potencial de desarrollo económico más frecuente o fácil de conseguir entre los considerados es el Índice de concentración sectorial, mientras que el que menos aporta o influye en el potencial de desarrollo económico dentro del conjunto elegido de 98 municipios por ser más difícil de superar dadas las habilidades de los sujetos es Ingresos presupuestarios por habitante.

En el análisis de la unidimensionalidad en ambas mediciones se detectan desviaciones respecto a los valores que Linacre define como óptimos en su regla de oro, por lo que el modelo detecta ciertas tensiones multidimensionales en el constructo. No obstante, para ratificar que las medidas sólo están influidas por una sola dimensión, se emplea de manera complementaria la evaluación de los desajustes de sujetos e ítems mediante los estadísticos Infit y Outfit. En este sentido, se ha de recordar que, si éstos presentan valores muy desajustados, las medidas podrían estar influidas por una segunda dimensión. Sin embargo, los estadísticos Infit y Outfit no presentan valores desajustados a nivel global, muy próximos a 1 en su MNSQ y entre 0.0 y -0.1 en el ZSTD tanto en sujetos como en ítems, en ambas mediciones, pudiéndose intuir que las medidas no están influidas por otras dimensiones, así como aceptar por ello 
que, con la información disponible, los datos solamente estarán influidos por una única dimensión a la hora de realizar cualquier análisis.

Respecto al análisis de la validez y la fiabilidad global, para el caso de la separación de los ítems alcanza 0.98 y en los sujetos 0.39 . Cuanto más alto sea el valor obtenido, mejor es la separación existente y más precisa es la medición; pero también se ha de mencionar que Oreja (2005) considera que los mínimos de fiabilidad de referencia aceptables ampliamente difundidos no han sido nunca justificados teóricamente, mientras que otros estudios han analizado las distintas condiciones que determinan los niveles de fiabilidad: la muestra, el tipo de muestra, el tipo de escala, el número de categorías en las escalas de ítems, el número de ítems en las escalas, el número de ítems suprimidos, la naturaleza del constructo, el tipo de investigación, etc. En definitiva, un determinado nivel de fiabilidad per se no puede considerarse alto o bajo, ya que para ello es necesario considerarlo conjuntamente con las características peculiares del estudio en cuestión, es decir, debe ser tenido en cuenta junto a las características propias de los trabajos de investigación llevados a cabo, en este caso dentro del ámbito económico y empresarial, debiéndose tener siempre presente este marco de referencia para la correcta interpretación del nivel de fiabilidad obtenido.

En este sentido, también se ha de poner de manifiesto como Stenner (1994) señala, la diferencia entre la objetividad absoluta más propia de las ciencias físicas y químicas y la objetividad específica desarrollada por Rasch, fruto esta última del ajuste de un conjunto de datos al modelo de Rasch. Así, la posición relativa de los parámetros de los sujetos y de los ítems en el continuo lineal del constructo es independiente de sus muestras; en cambio, las medidas absolutas se pueden conseguir de manera indirecta incluyendo ítems o personas de referencia en el análisis. Por tanto, la delimitación de los sujetos, su grado de homogeneidad, el comportamiento dispar ligado al tiempo o al espacio, así como las posibilidades de los mismos para intervenir de forma no normalizada o disonante respecto a los demás, puede dar lugar no a restar objetividad específica, pero sí a marcar mayor grado de ligazón entre sujetos y medición de la variable latente en torno a unos ítems concretos, afectando a unos resultados más ligados al momento, al lugar o a otras variables no incluidas en la medición por no formar parte de la variable latente (aunque sí del sujeto).

En este punto, y a partir de la Tabla 3, se estudia de manera más detallada el posicionamiento jerárquico de los sujetos. El PTMEASURE-A presenta valores positivos en prácticamente todos los sujetos en ambas mediciones, lo que es indicativo de que tienen un comportamiento acorde al resto de los sujetos, si bien 4 municipios en el caso de la medición de 2007 y 5 en la de 2012 muestran un valor negativo, lo que refleja un comportamiento contrario al del resto de municipios. Tal es el caso de Aznalcóllar en 2007 o de Marinaleda en 2012, si bien hemos preferido no eliminar del estudio dichos municipios para su estudio.

Se ha de destacar que tanto para el año 2007 como para el año 2012, el líder en potencial de desarrollo económico dentro de los 98 municipios seleccionados es Espartinas, mientras que los municipios peor posicionados son Isla Mayor y Castilblanco de los Arroyos en 2007, y El Cuervo de Sevilla y Villanueva del Río y Minas en 2012.

Respecto a los estadísticos de validez, se ha de decir que en la Tabla 3 de medidas y ajustes se pueden ver los municipios con desajustes a través de los estadísticos Infit/Outfit. En este sentido, se ha de recordar que en la MNSQ del Infit/Outfit, el valor esperado es 1, admitiéndose como aceptables valores entre 0'5 y 1'5. Valores superiores a 1'5 e inferiores a 2 indican desajustes que son improductivos para la construcción de la medida, pero no distorsionan, mientras que valores superiores a 2, indican desajustes que sí distorsionan. Por otro lado, en la ZSTD del Infit/Outfit, los valores incluidos dentro del rango -1.9 y +1.9 son aceptables, mientras que los valores superiores a 2 están desajustados (Oreja, 2005: 70). 
Tabla 3. Medidas y ajustes de los sujetos para la medición de los municipios de la provincia de Sevilla (2007 y 2012$)$.

\begin{tabular}{|c|c|c|c|c|c|c|c|c|c|c|c|c|c|c|c|c|c|c|c|}
\hline \multirow[b]{2}{*}{$\begin{array}{c}\text { TOTA } \\
\text { L } \\
\end{array}$} & \multirow[b]{2}{*}{$\begin{array}{c}\text { MEASUR } \\
\text { E }\end{array}$} & \multirow{2}{*}{$\begin{array}{l}\text { MODE } \\
\text { L } \\
\text { S.E. }\end{array}$} & \multicolumn{2}{|c|}{ INFIT } & \multicolumn{2}{|c|}{ OUTFIT } & \multicolumn{2}{|c|}{$\begin{array}{l}\text { PTMEASURE- } \\
\text { A }\end{array}$} & \multicolumn{2}{|c|}{ PERSON } & \multirow{2}{*}{$\begin{array}{c}\text { TOTA } \\
\text { L } \\
\text { SCOR } \\
\text { E }\end{array}$} & \multirow[b]{2}{*}{$\begin{array}{c}\text { MEASUR } \\
\mathrm{E}\end{array}$} & \multirow{2}{*}{$\begin{array}{l}\text { MODE } \\
\text { L.E. }\end{array}$} & \multicolumn{2}{|c|}{ INFIT } & \multicolumn{2}{|c|}{ OUTFIT } & \multicolumn{2}{|c|}{$\begin{array}{l}\text { PTMEASURE- } \\
\text { A }\end{array}$} \\
\hline & & & $\begin{array}{c}\mathrm{MNS} \\
\mathrm{Q}\end{array}$ & $\begin{array}{c}\text { ZST } \\
\text { D }\end{array}$ & $\begin{array}{c}\text { MNS } \\
Q\end{array}$ & $\begin{array}{c}\text { ZST } \\
\text { D }\end{array}$ & CORR. & EXP & 2007 & 2012 & & & & $\begin{array}{c}\text { MNS } \\
Q\end{array}$ & $\begin{array}{c}\text { ZST } \\
\text { D }\end{array}$ & $\begin{array}{l}\text { MNS } \\
Q\end{array}$ & $\begin{array}{c}\text { ZST } \\
\text { D }\end{array}$ & CORR. & EXP. \\
\hline 47 & .72 & .22 & 1.11 & .4 & .94 & .2 & .60 & .55 & 40-Espartinas & 40-Espartinas & 44 & .46 & .19 & 1.45 & .9 & 1.33 & .7 & .50 & .48 \\
\hline 42 & .50 & .20 & 1.42 & .9 & 1.21 & .5 & .70 & .61 & 91-Tomares & 91-Tomares & 43 & .43 & .19 & 1.55 & 1.1 & 1.40 & .8 & .53 & .49 \\
\hline 41 & .45 & .20 & 1.69 & 1.3 & 1.43 & .8 & .67 & .62 & 70-Palomares del Río & 54-Lora de Estepa & 42 & .39 & .18 & .49 & -1.1 & .50 & -.9 & .65 & .50 \\
\hline 40 & .41 & .20 & 1.67 & 1.2 & 1.90 & 1.4 & .19 & .62 & 11-Aznalcázar & 94-Valencina de la Concepción & 41 & .36 & .18 & 1.45 & 1.0 & 1.32 & .7 & .62 & .51 \\
\hline 40 & .41 & .20 & 2.01 & 1.7 & 1.70 & 1.2 & .65 & .62 & 27-Castilleja de Guzmán & 1-Aguadulce & 40 & .32 & .18 & 1.03 & .2 & 1.05 & .3 & .59 & .52 \\
\hline 39 & .37 & .20 & .79 & -.3 & .70 & -.4 & .72 & .63 & 15-Bollullos de la Mitación & 41-Estepa & 40 & .32 & .18 & .33 & -1.7 & .32 & -1.6 & .88 & .52 \\
\hline 39 & .37 & .20 & 1.44 & .9 & 1.31 & .7 & .76 & .63 & 47-Gines & 59-Mairena del Aljarafe & 40 & .32 & .18 & 1.57 & 1.1 & 1.45 & .9 & .60 & .52 \\
\hline 39 & .37 & .20 & 1.05 & .3 & .96 & .1 & .74 & .63 & 59-Mairena del Aljarafe & 70-Palomares del Río & 40 & .32 & .18 & 1.92 & 1.6 & 1.75 & 1.3 & .54 & .52 \\
\hline 39 & .37 & .20 & 1.16 & .5 & 1.09 & .4 & .53 & .63 & 92-Umbrete & 90-Sevilla (capital) & 40 & .32 & .18 & 1.23 & .6 & 1.14 & .4 & .61 & .52 \\
\hline 39 & .37 & .20 & 1.29 & .7 & 1.20 & .5 & .75 & .63 & 94-Valencina de la Concepción & 27-Castilleja de Guzmán & 39 & .29 & .18 & 2.22 & 2.0 & 2.04 & 1.7 & .51 & .53 \\
\hline 38 & .33 & .20 & 1.39 & .8 & 1.37 & .8 & .53 & .63 & 1-Aguadulce & 47-Gines & 39 & .29 & .18 & 1.92 & 1.6 & 1.78 & 1.4 & .55 & .53 \\
\hline 38 & .33 & .20 & .43 & -1.2 & .38 & -1.3 & .83 & .63 & 29-Castilleja del Campo & 50-Herrera & 39 & .29 & .18 & .46 & -1.2 & .53 & -.9 & .65 & .53 \\
\hline 37 & .29 & .20 & .92 & .0 & .87 & .0 & .83 & .64 & 9-Almensilla & 15-Bollullos de la Mitación & 38 & .26 & .18 & 1.13 & .4 & 1.05 & .3 & .71 & .54 \\
\hline 37 & .29 & .20 & 1.93 & 1.6 & 1.73 & 1.3 & .63 & .64 & 44-Gelves & 34-Coripe & 38 & .26 & .18 & 1.09 & .4 & 1.34 & .8 & .28 & .54 \\
\hline 37 & .29 & .20 & .74 & -.3 & .67 & -.5 & .76 & .64 & 84-Salteras & 77-Puebla de los Infantes (La) & 38 & .26 & .18 & 1.82 & 1.5 & 2.16 & 1.9 & .07 & .54 \\
\hline 37 & .29 & .20 & 1.44 & .9 & 1.29 & .7 & .70 & .64 & 90-Sevilla (capital) & 7-Algámitas & 37 & .22 & .18 & 1.17 & .5 & 1.20 & .5 & .53 & .54 \\
\hline 36 & .25 & .20 & 1.94 & 1.6 & 1.78 & 1.3 & .68 & .64 & 16-Bormujos & 11-Aznalcázar & 37 & .22 & .18 & 91 & .0 & .85 & -.1 & .51 & .54 \\
\hline 36 & .25 & .20 & .38 & -1.4 & .40 & -1.2 & .81 & .64 & 50 -Herrera & 16-Bormujos & 37 & .22 & .18 & 1.57 & 1.1 & 1.50 & 1.0 & .64 & .54 \\
\hline 36 & .25 & .20 & .35 & -1.5 & .35 & -1.4 & .91 & .64 & 54-Lora de Estepa & 22-Cañada Rosal & 37 & .22 & .18 & .64 & -.6 & .66 & -.6 & .68 & .54 \\
\hline 35 & .21 & .20 & .99 & .2 & .85 & -.1 & .66 & .64 & 41-Estepa & 44-Gelves & 37 & .22 & .18 & 2.11 & 1.9 & 1.98 & 1.7 & .55 & .54 \\
\hline 35 & .21 & .20 & .85 & -.1 & .90 & .0 & .61 & .64 & $\begin{array}{c}\text { 66-Navas de la Concepción } \\
\text { (Las) }\end{array}$ & 46-Gilena & 37 & .22 & .18 & .33 & -1.7 & .30 & -1.7 & .69 & .54 \\
\hline 34 & .17 & .20 & .62 & -.6 & .62 & -.6 & .87 & .64 & 22-Cañada Rosal & 57-Madroño (El) & 37 & .22 & .18 & 3.51 & 3.3 & 4.53 & 3.9 & -.51 & .54 \\
\hline 34 & .17 & .20 & .31 & -1.6 & .27 & -1.7 & .85 & .64 & 46-Gilena & 5-Alcolea del Río & 36 & .19 & .18 & 1.86 & 1.5 & 1.78 & 1.4 & .24 & .55 \\
\hline 33 & .13 & .20 & .06 & -3.2 & .07 & -3.0 & .94 & .64 & 4-Alcalá del Río & 36-Corrales (Los) & 36 & .19 & .18 & .87 & -.1 & .93 & .0 & .54 & .55 \\
\hline 33 & .13 & .20 & .22 & -2.0 & .21 & -2.0 & .83 & .64 & 14-Benacazón & 68-Osuna & 36 & .19 & .18 & .20 & -2.3 & .24 & -2.0 & .71 & .55 \\
\hline 33 & .13 & .20 & .49 & -1.0 & .52 & -.9 & .60 & .64 & 82-Ronquillo (El) & 83-Rubio (El) & 35 & .16 & .18 & .93 & .0 & .96 & .1 & .58 & .55 \\
\hline 32 & .09 & .20 & .92 & .0 & .87 & .0 & .80 & .64 & 36-Corrales (Los) & 42-Fuentes de Andalucía & 34 & .13 & .18 & .71 & -.5 & .67 & -.5 & .78 & .55 \\
\hline 32 & .09 & .20 & .95 & .1 & .86 & -.1 & .75 & .64 & 42-Fuentes de Andalucía & $\begin{array}{c}\text { 66-Navas de la Concepción } \\
\text { (Las) }\end{array}$ & 34 & .13 & .18 & .94 & .1 & 1.00 & .2 & .47 & .55 \\
\hline 32 & .09 & .20 & .62 & -.6 & .59 & -.7 & .81 & .64 & 56-Luisiana (La) & 73-Peñaflor & 34 & .13 & .18 & 1.55 & 1.1 & 1.43 & .9 & .32 & .55 \\
\hline 32 & .09 & .20 & .25 & -1.9 & .23 & -1.9 & .85 & .64 & 68-Osuna & 84-Salteras & 34 & .13 & .18 & .76 & -.3 & .75 & -.4 & .75 & .55 \\
\hline 31 & .05 & .20 & 1.02 & .2 & .88 & .0 & .67 & .63 & 3-Alcalá de Guadaíra & 9-Almensilla & 33 & .09 & .18 & 1.21 & .6 & 1.23 & .6 & .75 & .56 \\
\hline 31 & .05 & .20 & .89 & .0 & .85 & -.1 & .53 & .63 & 48-Guadalcanal & 29-Castilleja del Campo & 33 & .09 & .18 & .88 & -.1 & 1.00 & .2 & .12 & .56 \\
\hline 31 & .05 & .20 & 3.79 & 3.3 & 5.07 & 4.0 & -.41 & .63 & 57-Madroño (El) & 81-Roda de Andalucía (La) & 33 & .09 & .18 & .67 & -.5 & .65 & -.6 & .36 & .56 \\
\hline 31 & .05 & .20 & .96 & .1 & .87 & .0 & .84 & .63 & 58-Mairena del Alcor & 89-Saucejo (El) & 33 & .09 & .18 & .62 & -.7 & .63 & -.6 & .76 & .56 \\
\hline
\end{tabular}




\begin{tabular}{|c|c|c|c|c|c|c|c|c|c|c|c|c|c|c|c|c|c|c|c|}
\hline 31 & .05 & .20 & 1.50 & 1.0 & 1.70 & 1.2 & .34 & .63 & 61-Marinaleda & 2-Alanís & 32 & .06 & .18 & 1.26 & .6 & 1.33 & .8 & .16 & .56 \\
\hline 30 & .01 & .20 & 1.22 & .6 & 1.23 & .6 & .43 & .63 & 7-Algámitas & 25-Casariche & 32 & .06 & .18 & .38 & -1.4 & .39 & -1.4 & .67 & .56 \\
\hline 30 & .01 & .20 & 1.11 & .4 & .97 & .1 & .49 & .63 & 13-Badolatosa & 31-Cazalla de la Sierra & 32 & .06 & .18 & .15 & -2.5 & .16 & -2.4 & .84 & .56 \\
\hline 30 & .01 & .20 & .53 & -.8 & .48 & -.9 & .72 & .63 & 23-Carmona & 61-Marinaleda & 32 & .06 & .18 & 1.97 & 1.6 & 2.05 & 1.7 & -.04 & .56 \\
\hline 30 & .01 & .20 & .31 & -1.6 & .27 & -1.6 & .85 & .63 & 24-Carrión de los Céspedes & 64-Montellano & 32 & .06 & .18 & .47 & -1.1 & .51 & -1.0 & .62 & .56 \\
\hline 30 & .01 & .20 & 1.21 & .5 & 1.12 & .4 & .75 & .63 & 38-Dos Hermanas & 13-Badolatosa & 31 & .03 & .18 & 2.36 & 2.1 & 2.38 & 2.1 & -.12 & .56 \\
\hline 30 & .01 & .20 & .29 & -1.6 & .29 & -1.6 & .90 & .63 & 39-Écija & 55-Lora del Río & 31 & .03 & .18 & 1.35 & .8 & 1.23 & .6 & .34 & .56 \\
\hline 30 & .01 & .20 & .50 & -.9 & .51 & -.9 & .85 & .63 & 83-Rubio (El) & 39-Écija & 30 & -.01 & .19 & .56 & -.8 & .58 & -.7 & .71 & .56 \\
\hline 30 & .01 & .20 & 1.58 & 1.1 & 1.40 & .8 & .81 & .63 & 88-Santiponce & 56-Luisiana (La) & 30 & -.01 & .19 & .24 & -2.0 & .22 & -2.0 & .80 & .56 \\
\hline 30 & .01 & .20 & .67 & -.5 & .63 & -.5 & .85 & .63 & 89-Saucejo (El) & 65-Morón de la Frontera & 30 & -.01 & .19 & .60 & -.7 & .60 & -.7 & .62 & .56 \\
\hline 29 & -.03 & .21 & .76 & -.3 & .76 & -.2 & .66 & .62 & 2-Alanís & 71-Paradas & 30 & -.01 & .19 & .24 & -2.0 & .25 & -1.9 & .84 & .56 \\
\hline 29 & -.03 & .21 & 2.05 & 1.7 & 1.82 & 1.3 & .31 & .62 & 5-Alcolea del Río & 82-Ronquillo (El) & 30 & $\begin{array}{l}-.01 \\
\end{array}$ & .19 & .48 & -1.1 & .55 & -.8 & .55 & .56 \\
\hline 29 & -.03 & .21 & .32 & -1.5 & .27 & -1.6 & .78 & .62 & 35-Coronil (El) & $\begin{array}{l}\text { 95-Villamanrique de la } \\
\text { Condesa }\end{array}$ & 30 & -.01 & .19 & 1.98 & 1.6 & 1.79 & 1.4 & .14 & .56 \\
\hline 29 & -.03 & .21 & .39 & -1.3 & .38 & -1.2 & .72 & .62 & 49-Guillena & 38-Dos Hermanas & 29 & -.04 & .19 & .97 & .1 & 1.00 & .2 & .78 & .55 \\
\hline 29 & -.03 & .21 & .65 & -.5 & .63 & -.5 & .63 & .62 & 55-Lora del Río & 45-Gerena & 29 & -.04 & .19 & .50 & -1.0 & .51 & -.9 & .71 & .55 \\
\hline 29 & -.03 & .21 & .53 & -.9 & .49 & -.9 & .70 & .62 & 71-Paradas & 48-Guadalcanal & 29 & -.04 & .19 & .61 & -.7 & .62 & -.6 & .41 & .55 \\
\hline 29 & -.03 & .21 & 1.85 & 1.4 & 1.66 & 1.1 & .36 & .62 & 77-Puebla de los Infantes (La) & 58-Mairena del Alcor & 29 & -.04 & .19 & .94 & .1 & .92 & .0 & .84 & .55 \\
\hline 29 & -.03 & .21 & .24 & -1.9 & .22 & -1.8 & .82 & .62 & 80-Rinconada (La) & 75-Pruna & 29 & -.04 & .19 & 2.14 & 1.8 & 2.16 & 1.8 & .18 & .55 \\
\hline 29 & -.03 & .21 & .51 & -.9 & .51 & -.8 & .72 & .62 & 81-Roda de Andalucía (La) & 92-Umbrete & 29 & -.04 & .19 & .65 & -.6 & .65 & -.5 & .87 & .55 \\
\hline 29 & -.03 & .21 & .65 & -.5 & .62 & -.6 & .74 & .62 & 87-Sanlúcar la Mayor & 3-Alcalá de Guadaíra & 28 & -.08 & .19 & .68 & -.5 & .75 & -.3 & .58 & .55 \\
\hline 28 & -.07 & .21 & .91 & .0 & .69 & -.4 & .63 & .61 & 34-Coripe & 23-Carmona & 28 & -.08 & .19 & .30 & -1.7 & .28 & -1.7 & .86 & .55 \\
\hline 28 & -.07 & .21 & 3.69 & 3.1 & 5.57 & 4.1 & -.42 & .61 & 43-Garrobo (El) & 24-Carrión de los Céspedes & 28 & -.08 & .19 & .46 & -1.1 & .40 & -1.2 & .65 & .55 \\
\hline 28 & -.07 & .21 & .81 & -.2 & .68 & -.4 & .71 & .61 & 65-Morón de la Frontera & 60-Marchena & 28 & -.08 & .19 & .22 & -2.0 & .21 & -2.0 & .84 & .55 \\
\hline 28 & -.07 & .21 & .13 & -2.5 & .17 & -2.0 & .94 & .61 & 69-Palacios y Villafranca (Los) & 76-Puebla de Cazalla (La) & 28 & -.08 & .19 & .32 & -1.6 & .33 & -1.5 & .95 & .55 \\
\hline 28 & -.07 & .21 & .70 & -.4 & .61 & -.6 & .60 & .61 & 74-Pilas & 4-Alcalá del Río & 27 & -.12 & .19 & .31 & -1.6 & .26 & -1.7 & .80 & .55 \\
\hline 28 & -.07 & .21 & .54 & -.8 & .59 & -.6 & .82 & .61 & 76-Puebla de Cazalla (La) & 10-Arahal & 27 & -.12 & .19 & .37 & -1.4 & .36 & -1.3 & .72 & .55 \\
\hline 27 & -.12 & .21 & .34 & -1.4 & .34 & -1.3 & .79 & .60 & 10-Arahal & 14-Benacazón & 27 & -.12 & .19 & .24 & -1.9 & .31 & -1.5 & .91 & .55 \\
\hline 27 & -.12 & .21 & 2.17 & 1.8 & 2.20 & 1.7 & .24 & .60 & 17-Brenes & 17-Brenes & 27 & -.12 & .19 & 1.85 & 1.4 & 1.63 & 1.1 & .20 & .55 \\
\hline 27 & -.12 & .21 & .12 & -2.5 & .15 & -2.1 & .91 & .60 & 25-Casariche & 53-Lebrija & 27 & -.12 & .19 & .60 & -.7 & .67 & -.5 & .52 & .55 \\
\hline 27 & -.12 & .21 & 2.86 & 2.4 & 2.44 & 1.9 & .15 & .60 & $\begin{array}{l}\text { 95-Villamanrique de la } \\
\text { Condesa }\end{array}$ & 62-Martín de la Jara & 27 & -.12 & .19 & 2.72 & 2.4 & 2.56 & 2.1 & -.06 & .55 \\
\hline 26 & -.16 & .21 & 1.04 & .3 & 1.00 & .2 & .76 & .59 & 19-Camas & 87-Sanlúcar la Mayor & 27 & -.12 & .19 & .55 & -.8 & .57 & -.7 & .75 & .55 \\
\hline 26 & -.16 & .21 & 1.16 & .5 & .92 & .1 & .48 & .59 & 21-Cantillana & 19-Camas & 26 & -.15 & .19 & .67 & -.5 & .70 & -.4 & .81 & .54 \\
\hline 26 & -.16 & .21 & 1.04 & .3 & 1.00 & .2 & .76 & .59 & 28-Castilleja de la Cuesta & 20-Campana (La) & 26 & -.15 & .19 & 2.29 & 1.9 & 2.35 & 1.9 & .08 & .54 \\
\hline 26 & -.16 & .21 & .24 & -1.8 & .21 & -1.8 & .94 & .59 & 31-Cazalla de la Sierra & 28-Castilleja de la Cuesta & 26 & -.15 & .19 & .72 & -.4 & .84 & -.1 & .69 & .54 \\
\hline 26 & -.16 & .21 & .79 & -.2 & .68 & -.4 & .71 & .59 & 45-Gerena & 72-Pedroso (El) & 26 & -.15 & .19 & .73 & -.4 & .94 & .1 & .54 & .54 \\
\hline 26 & -.16 & .21 & .25 & -1.8 & .28 & -1.5 & .83 & .59 & 64-Montellano & 80-Rinconada (La) & 25 & -.19 & .20 & .60 & -.6 & .62 & -.5 & .74 & .53 \\
\hline 26 & -.16 & .21 & .13 & -2.4 & .12 & -2.3 & .89 & .59 & 72-Pedroso (El) & 88-Santiponce & 25 & -.19 & .20 & 1.02 & .2 & .99 & .2 & .77 & .53 \\
\hline 25 & -.21 & .22 & 1.70 & 1.2 & 2.60 & 2.0 & -.12 & .58 & 12-Aznalcóllar & 12-Aznalcóllar & 24 & -.23 & .20 & .92 & .0 & .74 & -.3 & .76 & .53 \\
\hline 25 & -.21 & .22 & 2.56 & 2.1 & 2.26 & 1.7 & .16 & .58 & 20-Campana (La) & 8-Almadén de la Plata & 23 & -.27 & .21 & 1.02 & .2 & 1.11 & .4 & .49 & .52 \\
\hline
\end{tabular}




\begin{tabular}{|c|c|c|c|c|c|c|c|c|c|c|c|c|c|c|c|c|c|c|c|}
\hline 25 & -.21 & .22 & 1.13 & .4 & 1.06 & .3 & .47 & .58 & 52-Lantejuela (La) & 30-Castillo de las Guardas (El) & 23 & -.27 & .21 & 1.14 & .4 & 1.16 & .5 & .55 & .52 \\
\hline 25 & -.21 & .22 & .15 & -2.3 & .15 & -2.0 & .91 & .58 & 53-Lebrija & 32-Constantina & 23 & -.27 & .21 & .76 & -.2 & .77 & -.2 & .46 & .52 \\
\hline 25 & -.21 & .22 & .89 & .0 & .92 & .1 & .74 & .58 & 85-San Juan de Aznalfarache & 35-Coronil (El) & 23 & -.27 & .21 & .62 & -.6 & .47 & -.9 & .39 & .52 \\
\hline 24 & -.26 & .22 & .24 & -1.7 & .33 & -1.2 & .84 & .57 & 18-Cabezas de San Juan (Las) & 49-Guillena & 23 & -.27 & .21 & .99 & .2 & .98 & .2 & .60 & .52 \\
\hline 24 & -.26 & .22 & .45 & -1.0 & .56 & -.6 & .89 & .57 & 33-Coria del Río & 51-Isla Mayor & 23 & -.27 & .21 & 1.83 & 1.3 & 1.91 & 1.4 & .10 & .52 \\
\hline 24 & -.26 & .22 & .58 & -.7 & .55 & -.6 & .79 & .57 & 60-Marchena & 74-Pilas & 23 & -.27 & .21 & .27 & -1.6 & .27 & -1.5 & .60 & .52 \\
\hline 24 & -.26 & .22 & 1.65 & 1.1 & 1.53 & .9 & .22 & .57 & 73-Peñaflor & 79-Real de la Jara (El) & 23 & -.27 & .21 & .74 & -.3 & 1.07 & .3 & .39 & .52 \\
\hline 24 & -.26 & .22 & 1.05 & .3 & 1.15 & .4 & .50 & .57 & 75-Pruna & 86-San Nicolás del Puerto & 23 & -.27 & .21 & 1.72 & 1.2 & 2.10 & 1.6 & -.37 & .52 \\
\hline 24 & -.26 & .22 & .34 & -1.4 & .46 & -.8 & .64 & .57 & 79-Real de la Jara (El) & 93-Utrera & 23 & -.27 & .21 & .33 & -1.4 & .39 & -1.1 & .82 & .52 \\
\hline 24 & -.26 & .22 & .58 & -.7 & .58 & -.5 & .71 & .57 & 93-Utrera & 98-Viso del Alcor (El) & 23 & -.27 & .21 & .47 & -1.0 & .44 & -.9 & .90 & .52 \\
\hline 23 & -.31 & .23 & 2.12 & 1.6 & 1.77 & 1.2 & .21 & .55 & 62-Martín de la Jara & 85-San Juan de Aznalfarache & 22 & -.32 & .21 & .59 & -.6 & .74 & -.2 & .57 & .51 \\
\hline 23 & -.31 & .23 & .78 & -.2 & .74 & -.2 & .77 & .55 & 67-Olivares & 18-Cabezas de San Juan (Las) & 21 & -.36 & .22 & .64 & -.5 & .70 & -.3 & .59 & .50 \\
\hline 23 & -.31 & .23 & 1.42 & .8 & 1.32 & .7 & .26 & .55 & 97-Villaverde del Río & 21-Cantillana & 21 & -.36 & .22 & .80 & -.1 & .67 & -.3 & .55 & .50 \\
\hline 23 & -.31 & .23 & .60 & -.6 & .53 & -.7 & .81 & .55 & 98-Viso del Alcor (El) & 69-Palacios y Villafranca (Los) & 21 & -.36 & .22 & .50 & -.8 & .59 & -.5 & .65 & .50 \\
\hline 22 & -.36 & .23 & .83 & -.1 & .77 & -.1 & .72 & .54 & 6-Algaba (La) & 97-Villaverde del Río & 21 & -.36 & .22 & .75 & -.2 & .69 & -.3 & .35 & .50 \\
\hline 22 & -.36 & .23 & .89 & .0 & 1.31 & .6 & .29 & .54 & 30-Castillo de las Guardas (El) & 33-Coria del Río & 20 & -.41 & .22 & .92 & .1 & .85 & .0 & .57 & .48 \\
\hline 21 & -.42 & .24 & .80 & -.1 & .95 & .2 & .59 & .52 & 32-Constantina & 52-Lantejuela (La) & 20 & -.41 & .22 & .86 & .0 & .97 & .2 & .51 & .48 \\
\hline 20 & -.48 & .25 & .30 & -1.3 & .23 & -1.4 & .76 & .50 & 37-Cuervo de Sevilla (El) & 6-Algaba (La) & 19 & -.47 & .23 & .58 & -.5 & .62 & -.4 & .69 & .47 \\
\hline 20 & -.48 & .25 & .17 & -1.8 & .30 & -1.2 & .92 & .50 & 63-Molares (Los) & 26-Castilblanco de los Arroyos & 19 & -.47 & .23 & 1.50 & .9 & 1.59 & 1.0 & .42 & .47 \\
\hline 20 & -.48 & .25 & 1.60 & 1.0 & 1.13 & .4 & .66 & .50 & 78-Puebla del Río (La) & 67-Olivares & 19 & -.47 & .23 & .73 & -.2 & .57 & -.5 & .78 & .47 \\
\hline 20 & -.48 & .25 & 2.99 & 2.2 & 3.21 & 2.3 & -.65 & .50 & 86-San Nicolás del Puerto & 63-Molares (Los) & 18 & -.52 & .24 & .49 & -.7 & .48 & -.7 & .64 & .45 \\
\hline 19 & -.54 & .26 & 1.27 & .6 & 1.33 & .7 & .29 & .48 & 8-Almadén de la Plata & 43-Garrobo (El) & 17 & -.58 & .25 & 1.38 & .7 & 1.00 & .2 & .61 & .44 \\
\hline 18 & -.61 & .27 & 1.24 & .6 & .76 & -.1 & .46 & .46 & 96-Villanueva del Río y Minas & 78-Puebla del Río (La) & 17 & -.58 & .25 & 1.96 & 1.3 & 1.37 & .7 & .70 & .44 \\
\hline 17 & -.69 & .29 & 3.13 & 2.1 & 3.31 & 2.2 & .09 & .44 & 51-Isla Mayor & 37-Cuervo de Sevilla (El) & 15 & -.72 & .28 & 1.09 & .4 & .95 & .2 & .64 & .39 \\
\hline 16 & -.78 & .31 & 1.95 & 1.2 & 1.44 & .8 & .33 & .42 & 26-Castilblanco de los Arroyos & 96-Villanueva del Río y Minas & 14 & -.81 & .30 & .97 & .2 & .65 & -.2 & .64 & .37 \\
\hline 29.3 & -.03 & .21 & 1.03 & -.1 & 1.02 & -.1 & & & MEAN & MEAN & 29.5 & -.05 & .20 & 1.00 & .0 & 1.00 & .0 & & \\
\hline 6.1 & .27 & .02 & .77 & 1.3 & .89 & 1.2 & & & S.D. & S.D. & 7.0 & .27 & .02 & .64 & 1.2 & .67 & 1.1 & & \\
\hline
\end{tabular}

Fuente: Elaboración propia a partir de Winsteps 3.81.0. 
A continuación, se pretende conseguir otro de los objetivos de este estudio: determinar cuáles son las condiciones facilitadoras o factores impulsores del potencial de desarrollo económico de un municipio más y menos importantes.

En la Tabla 4 de medidas y ajustes se presentan los atributos o ítems seleccionados para estudiar su incidencia de cara a facilitar o impulsar el potencial de desarrollo económico de un municipio, ordenados de menor a mayor presencia en el marco de referencia elegido de 98 municipios, de acuerdo con la percepción o respuesta de los mismos. Según los datos de esta tabla, los resultados son claros. El PTMEASURE-A presenta valores positivos en todos los ítems en ambas mediciones, lo que es indicativo de que ninguno de ellos tiene un comportamiento contrario al resto de los ítems.

Según la respuesta de los municipios, en ambos años, el ítem con medida más alta y, como consecuencia, más difícil de superar o conseguir fue Ingresos presupuestarios por habitante, mientras que el ítem con una medida más baja, y, por tanto, más fácil de superar o conseguir fue el Índice de concentración sectorial. De este modo, en ambos años, el factor que más grado de influencia tiene a la hora de generar un diferencial positivo en términos de potencial de desarrollo económico en los municipios que logren alcanzar una categoría mayor es el ítem 3, Ingresos presupuestarios por habitante, con una medida de 0.53 y 0.33 unidades logit para los años 2007 y 2012, respectivamente; seguido de Rentas netas del trabajo/Población 16-64 años con 0.25 y 0.24 unidades logit para los años 2007 y 2012, de manera respectiva.

En lo que respecta a los ítems con menor capacidad para generar diferencias debido a que la mayoría de los municipios elegidos alcanzó en dicho ítem una categoría elevada, destaca el ítem 6, Índice de concentración sectorial, con una medida de -0.50 y -0.47 para 2007 y 2012, respectivamente.

Se ha de destacar que los dos ítems posicionados en las primeras posiciones y mencionados anteriormente, presentan valores reducidos de score total, concretamente, 295 y 372 en 2007, y 337 y 368 en 2012, sobre los 980 posibles. Esto significaría que dichos ítems son difíciles de lograr, que existen marcadas diferencias entre los distintos municipios, que son pocos los que consiguen alcanzar un nivel categórico importante en estos atributos y que, por tanto, pueden marcar un diferencial importante en el potencial de desarrollo económico municipal.

El tercer ítem en importancia en el año 2012 es Paro registrado/Población de 16 a 64 años, con una medida 0.11 unidades logit, mientras que en 2007 es Contratos registrados/ Población de 16 a 64 años con una medida 0.17 unidades logit.

En 2012, el cuarto ítem con mayor impacto para lograr mejorar la fortaleza económica es Contratos registrados/Población de 16 a 64 años, que alcanza una medida de Rasch de 0.01 unidades logit; mientras que en 2007 es Paro registrado/Población de 16 a 64 años con una medida -0.03 unidades logit.

Esta permuta en la posición de ambos ítems es acorde al cambio de una fase expansiva a una de recesión económica, es decir, los resultados en estos ítems ponen de manifiesto una evolución desfavorable como es lógico dado el escenario global de crisis económica que comienza en 2008.

El ítem situado en quinto lugar en ambos años es Número de declaraciones/Población de 16 y 64 años, de tal manera que la evolución ha sido positiva en la media del conjunto de municipios considerados, con un aumento medio entre ambos años de un $4.14 \%$.

Para finalizar, el ítem ubicado en última posición en ambos años, Índice de concentración sectorial, disminuyó en 2012 respecto a 2007 un 6.36\% de media. Puede observarse además cómo el score total de este ítem es 685 y 709 en 2007 y 2012, respectivamente, cifras muy cercanas ya al total disponible de 980 , lo que mostraría además el poco margen de dicho ítem como factor de incidencia diferencial en el potencial de desarrollo económico municipal. 
Tabla 4. Medidas y ajustes de los ítems para la medición de los municipios de la provincia de Sevilla (2007-2012).

\begin{tabular}{|c|c|c|c|c|c|c|c|c|c|c|c|c|c|}
\hline \multirow{2}{*}{$\begin{array}{c}\text { ENTRY } \\
\text { NUMBER }\end{array}$} & \multirow{2}{*}{\begin{tabular}{|l|} 
TOTAL \\
SCORE
\end{tabular}} & \multirow{2}{*}{$\begin{array}{l}\text { TOTAL } \\
\text { COUNT }\end{array}$} & \multirow[b]{2}{*}{ MEASURE } & \multirow{2}{*}{$\begin{array}{c}\text { MODEL } \\
\text { S.E. }\end{array}$} & \multicolumn{2}{|c|}{ INFIT } & \multicolumn{2}{|c|}{ OUTFIT } & \multicolumn{2}{|c|}{ PTMEASURE-A } & \multirow{2}{*}{$\begin{array}{c}\text { EXACT } \\
\text { OBS } \%\end{array}$} & \multirow{2}{*}{$\begin{array}{c}\text { МАТСН } \\
\text { ЕХР\% } \\
\end{array}$} & \multirow{2}{*}{ ÍTEM 2007} \\
\hline & & & & & \begin{tabular}{|l|l} 
MNSQ & 2 \\
\end{tabular} & ZSTD & MNSQ & ZSTD & CORR. & EXP. & & & \\
\hline 3 & 295 & \begin{tabular}{l|l}
98 &
\end{tabular} & .53 & \begin{tabular}{l|l}
.07 &
\end{tabular} & \begin{tabular}{|l|l}
1.04 \\
\end{tabular} & .3 & 1.18 & 1.0 & .28 & .38 & 38.8 & 27.6 & Ingresos presupuestarios por hab. \\
\hline 2 & 372 & 98 & .25 & .06 & .88 & -.8 & .73 & -1.8 & .66 & .44 & 27.6 & 21.4 & Rentas N. Trabajo/Pob. 16-64 años \\
\hline 4 & 396 & 98 & .17 & .05 & .83 & -1.2 & .74 & -1.8 & .23 & .46 & 29.6 & 20.4 & Contratos reg./Pob. 16-64 años \\
\hline 5 & 474 & 98 & -.03 & .05 & 1.58 & 3.9 & 1.71 & 4.4 & .23 & .49 & 9.2 & 17.2 & Paro registrado/Pob. 16-64 años \\
\hline 1 & 653 & 98 & -.43 & .05 & .93 & -.6 & .96 & -.3 & .76 & .50 & 10.2 & 16.3 & $\mathrm{~N}^{\mathrm{o}}$ declaraciones/Pob. 16-64 años \\
\hline 6 & 685 & 98 & -.50 & .05 & .79 & -1.7 & .79 & -1.6 & .49 & .50 & 23.5 & 16.1 & Índice de concentración sectorial \\
\hline MEAN & 479.2 & 98.0 & .00 & .05 & 1.01 & .0 & 1.02 & .0 & & & 23.1 & 19.8 & \\
\hline S.D. & 144.3 & .0 & .37 & .01 & .27 & 1.8 & .35 & 2.2 & & & 10.5 & 4.0 & \\
\hline \multirow{2}{*}{$\begin{array}{c}\text { ENTRY } \\
\text { NUMBER }\end{array}$} & \multirow{2}{*}{\begin{tabular}{|l|} 
TOTAL \\
SCORE
\end{tabular}} & \multirow{2}{*}{\begin{tabular}{|l} 
TOTAL \\
COUNT
\end{tabular}} & \multirow[b]{2}{*}{ MEASURE } & \multirow{2}{*}{$\begin{array}{c}\text { MODEL } \\
\text { S.E. }\end{array}$} & \multicolumn{2}{|c|}{ INFIT } & \multicolumn{2}{|c|}{ OUTFIT } & \multicolumn{2}{|c|}{ PTMEASURE-A } & & & \multirow[b]{2}{*}{ ÍTEM 2012} \\
\hline & & & & & MNSQ & ZSTD & $\begin{array}{c}\text { MNS } \\
\mathbf{Q}\end{array}$ & ZSTD & CORR. & EXP. & $\begin{array}{c}\text { EXACT } \\
\text { OBS } \%\end{array}$ & $\begin{array}{c}\text { МАТСН } \\
\text { ЕХР\% }\end{array}$ & \\
\hline 3 & 337 & 98 & .33 & .05 & .71 & -2.0 & .73 & -1.8 & .26 & .42 & 29.6 & 20.0 & Ingresos presupuestarios por hab. \\
\hline 2 & 368 & 98 & .24 & .05 & 1.14 & 1.0 & .97 & -.1 & .46 & .44 & 15.3 & 18.9 & Rentas N. Trabajo/Pob. 16-64 años \\
\hline 5 & 423 & 98 & .11 & .05 & 1.46 & 3.0 & 1.48 & 3.0 & .56 & .48 & 12.2 & 17.5 & Paro registrado/Pob. 16-64 años \\
\hline 4 & 468 & 98 & .01 & .05 & .88 & -1.0 & .83 & -1.3 & .36 & .50 & 21.4 & 16.4 & Contratos reg./Pob. 16-64 años \\
\hline 1 & 586 & 98 & -.22 & .04 & .77 & -2.0 & .79 & -1.7 & .84 & .53 & 9.2 & 15.1 & $\mathrm{~N}^{\mathrm{o}}$ declaraciones/Pob. 16-64 años \\
\hline 6 & 709 & 98 & -.47 & .05 & 1.10 & .8 & 1.19 & 1.3 & .19 & .52 & 10.2 & 14.3 & Índice de concentración sectorial \\
\hline MEAN & 481.8 & 98.0 & .00 & .05 & 1.01 & .0 & 1.00 & -.1 & & & 16.3 & 17.0 & \\
\hline S.D. & 129.1 & .0 & .28 & .00 & .26 & 1.8 & .26 & 1.8 & & & 7.2 & 2.0 & \\
\hline
\end{tabular}

Fuente: Elaboración propia a partir de Winsteps 3.81.0. 
Para estudiar de forma más detallada los municipios con desajustes en ambos años se puede emplear la información desagregada de la Tabla 5. En relación a la información contenida en la tabla mencionada, cabe recordar que en situaciones de análisis comparativo entre sujetos lo que se recoge es un desajuste que necesita ser explicado en caso de considerarse oportuno o necesario para el objeto del trabajo de investigación. Por ello mediante esta tabla se puede llevar a cabo un estudio detallado del origen del desajuste entre el valor real y el del modelo que permite ver en qué ítem se genera dicho desajuste. Se ha de recordar también que los valores residuales positivos indican que han puntuado al ítem por encima del valor que esperaba el modelo, mientras que los valores residuales negativos indican que han sido puntuados más bajo de lo que el modelo esperaba. Ello permite poner en marcha las acciones necesarias para corregir las causas del desajuste, en el caso de que se considere oportuno.

Tabla 5. Desajustes para los municipios de la provincia de Sevilla.

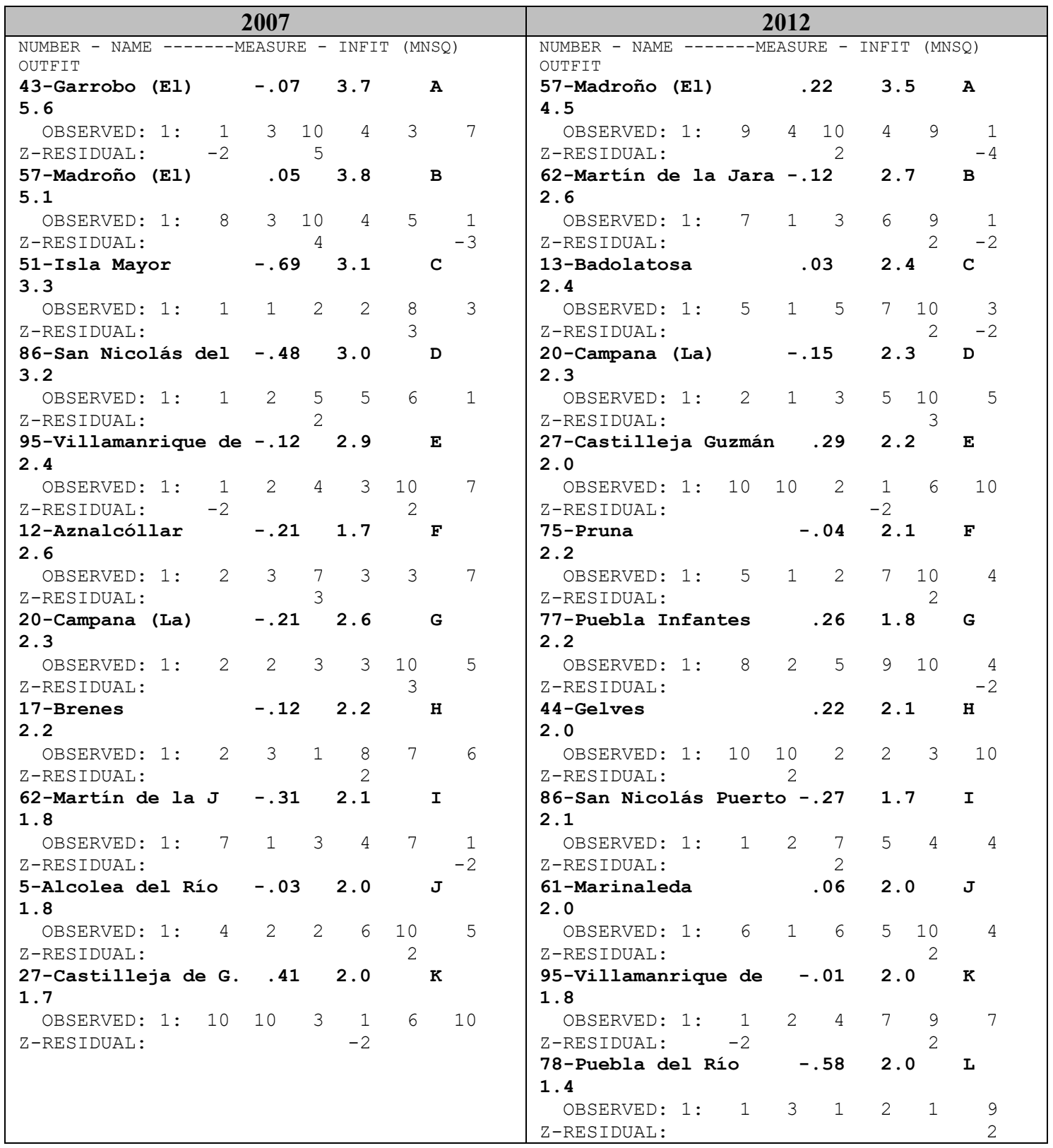

Fuente: Elaboración propia a partir de Winsteps 3.81.0. 
Por último, destacar que el modelo de Rasch permite también determinar qué ítems pueden llegar a desajustar. Sin embargo, en el caso en el que se trabaja ninguno presenta un desajuste relevante.

\section{Conclusiones.}

Definir el proceso de desarrollo económico de un territorio es de una enorme dificultad, puesto que implica la interacción de numerosos factores de diversa índole (económicos, sociales, culturales, institucionales...). La necesidad de realizar dicho proceso es evidente, dado que como consecuencia de ello se pueden diseñar medidas de política económica para influir en el mismo, generándolo o estimulando, redireccionándolo o potenciando el ya existente.

La presente investigación, basándose en la medición del Potencial de Desarrollo Económico obtenida mediante la aplicación del modelo de Rasch, ha logrado jerarquizar la posición de los municipios en función de esta variable latente y poner de manifiesto la relevancia o incidencia diferencial de los factores de potencialidad de dicho proceso y, en definitiva, la existencia de asimetrías entre los municipios, que expliquen la ordenación de los sujetos en relación al constructo elegido. Una vez medido, pueden definirse actuaciones y cambiar los resultados. Todo ello puede ayudar, sin lugar a dudas, al diseño más eficaz y eficiente de las políticas económicas dirigidas tanto a afianzar como a estimular el crecimiento y el desarrollo económico de las unidades municipales, dado que cada municipio sabe cuál es su situación de partida y qué ítems permiten avances más sencillos, pero menos impactantes, y cuáles más complejos, pero también más determinantes.

El análisis se desarrolla en unos escenarios constituidos por 98 sujetos (municipios de la provincia de Sevilla) en dos momentos diferentes, que abarcan un periodo de recesión económica importante, puesto que para este territorio el 2007 es el año con mayor crecimiento en su economía y el 2012 es el último año de recesión importante. Dada la entidad heterogénea existente, ofrece unos resultados que muestra la divergencia tanto en las características propias de cada territorio (económicas, sociales, culturales, institucionales...) como de nivel de desarrollo. Así, Espartinas y Tomares son los municipios que presiden la medición, los de mayor Potencial Económico, a pesar de que en el primero la distancia con la media de 2012 sea un $40.58 \%$ más baja que la de 2007 , mientras que para el segundo la distancia con su media en 2012 sea un 19.15\% menor que en 2007 (aunque trabajemos con dos mediciones específicas, y cada MPE de un municipio esté ligado a la propia medición, su distancia con la media del conjunto de municipios sí es comparable entre mediciones). Sin embargo, hemos podido comprobar cómo municipios como Peñaflor que ocupaba la $80^{\mathrm{a}}$ posición en 2007 , es el municipio que ha ganado más posiciones hasta 2012 (51 puestos), con un crecimiento de su MPE extraordinario, encontrándose muy por debajo de la media en 2007 para posicionarse por encima de la media en 2012. Le sigue la mejora experimentada por municipios como Coripe, La Puebla de los Infantes Cazalla de la Sierra o Montellano, entre otros. Por el lado contrario, Umbrete, El Garrobo, Benacazón o Alcalá del Río, entre otros pierden muchas posiciones en el ranking, desde 44 a 35 puestos.

En este contexto, el presente trabajo de investigación se presenta como un instrumento de análisis funcional, operativo, práctico, flexible, apropiado y eficiente para el estudio económico de los territorios a nivel municipal puesto que permite jerarquizar los sujetos seleccionados en función de su potencial de desarrollo económico, posibilitando incluso la ejecución de comparaciones intertemporales $\mathrm{y}$, en consecuencia, poder actuar con estrategias adecuadas.

Pero, además, permite ordenar los factores de potencialidad elegidos según su capacidad o relevancia para generar una incidencia diferencial en el citado proceso, siendo este último aspecto muy útil y valioso para la recomendación de estrategias de política económica capaces de estimular el desarrollo, teniendo en cuenta las características propias, así como las diferencias en el potencial del mismo de las distintas unidades territoriales. Al obtener qué ítems son los más incidentes en el proceso de Desarrollo Económico, según el periodo considerado, expansión o recesión económica, los municipios pueden lograr un mayor avance adecuando sus actuaciones estratégicas de política presupuestaria, laboral, empresarial y de dinamismo interno para movilizar renta y ser capaces así de 
crear más renta, riqueza y empleo. Así, en ambas mediciones los ingresos presupuestarios por habitante y las rentas netas de trabajo por población en edad de trabajar son los ítems que mayor impacto positivo puede causar, y son los más difíciles de conseguir, aunque su medición se ha reducido mucho en el caso del primero y apenas en el segundo. Los ítems más fáciles de obtener son la diversificación productiva y el número de declaraciones por población en edad de trabajar, y por lo tanto los de menor medida, que en ambos casos ha mejorado en 2012 respecto de 2007. Si un municipio no consigue un buen nivel en estos ítems de fácil consecución retrocederán de forma importante en la MPE.

Este análisis permite identificar los ítems fuertes y débiles en los que destaca cada municipio, lo que enfrentado a la ponderación que experimenta cada uno de éstos en la medición global nos facilita identificar cuál debe ser la estrategia específica de cada uno de ellos si desea mejorar su potencial económico.

Igualmente, los desajustes plantean la posibilidad de iniciar un estudio detallado de ciertos municipios que se salen de la tónica normal; así hay municipios que presentan un nivel de contratación excesivo a pesar de su carácter rural, lo que podría estar derivado de intervenciones específicas que provoquen tal circunstancia: hábitos locales, subvenciones específicas, etc. Es por ello que el modelo permite encontrar respuestas a ciertos desarrollos que requieren, lógicamente, de un análisis de tales escenarios de forma más amplia y pormenorizada.

Teniendo en cuenta los resultados obtenidos, se puede resaltar que se han logrado los objetivos planteados inicialmente: una propuesta analítica genérica que apoye la planificación de las políticas de desarrollo de un municipio, así como la confirmación de la eficacia tanto de dicha propuesta como de los instrumentos utilizados para los municipios analizados.

En relación a la consideración de las posibles limitaciones del presente trabajo de investigación, se podría destacar tanto las tensiones iniciales detectadas en el análisis de la unidimensionalidad del constructo en las dos evaluaciones de los municipios, como la mejorable fiabilidad global de los sujetos en la medición. Ambos hechos se deben, sin lugar a dudas, a la enorme heterogeneidad de los sujetos considerados en el escenario planteado y, en cualquier caso, no suponen un obstáculo para el análisis del objeto central del estudio, el potencial de desarrollo económico municipal.

Para finalizar, se pueden plantear algunas recomendaciones de cara a la posible realización de trabajos de investigación similares en un futuro en el citado ámbito municipal. En principio pretendemos hacer pruebas con mediciones que incorporen dos ítems adicionales en esta medición: uno que complemente el ítem de la diversificación/concentración productiva con la cantidad de tejido empresarial, es decir, el número de empresas por cada 1.000 habitantes; el segundo, sobre el número de oficinas bancarias por cada 1.000 habitantes. A partir de ello, realizaremos mediciones territoriales con grupos de municipios de tamaño poblacional similar, que favorezca una mayor coherencia de respuesta.

\section{Referencias}

Alvarado, J.M., \& Santisteban, C. (2006). La validez en la medición psicológica. Madrid: UNED.

Álvarez, P. (2008). El Modelo de Rasch como herramienta para obtener una única prioridad entre varias. En J. Febles Acosta (Coord.), Los Modelos de Rasch en Administración de Empresas. Aplicaciones Avanzadas (pp. 25-38). Santa Cruz de Tenerife: Fundación FYDE Caja Canarias.

Andersen, E.B. (1973). A goodness of fit test for the Rasch Model. Psychometrika, 38(1), 123-140.

Andrich, D. (1978). A rating formulation for ordered response categories. Psychometrika, 43, 357-374.

Andrich, D. (1988a). A General Form of Rasch's Extended Logistic Model for Partial Credit Scoring. Applied Measurement in Education, 1(4), 363-378. 
Andrich, D. (1988b). Rasch Models for measurement. Newbury Park, California: Sage.

Biehl, D. (1988). Las infraestructuras y el desarrollo regional. En E. Fuentes Quintana (Dir.), Papeles de Economía Española 35. Economía Regional: Ideas y Políticas (pp. 293-310). Madrid: Fundación Fondo para la Investigación Económica y Social - Obra Social de la Confederación Española de Cajas de Ahorros.

Caravaca, I., González, G., García, A., Fernández, V., \& Mendoza, A. (2014). Conocimiento, innovación y estrategias públicas de desarrollo: análisis comparado de tres ciudades medias de Andalucía. Revista Latinoamericana de Estudios Urbano Regionales (EURE), 40(119), 49-74.

Cliff, N. (1992). Abstract measurement theory and the revolution that never happened. Psychological Science, 3(3), 186-190.

Cuenca, E., Navarro, M., \& Morán, J. C. (2019). Factors determining differences in the poverty degree among countries. Mdpi, Resources, 8(3), 122.

De Haro, R., Marceleño, S., Irán, J., \& Nájera, O. (2017). Las desigualdades socioeconómicas entre los municipios de Nayarit, México. Revista Mexicana de Ciencias Políticas y Sociales, 230 (mayoagosto de 2017), 117-154.

Entrena-Durán, F., \& Álvarez-Lorente, T. (2014). Dinámicas sociodemográficas, economía y potencialidades de desarrollo sostenible de la Comarca de Guadix (España). Ambiente y Desarrollo, 18(35), 9-21.

Esparcia, J., Escribano, J., \& Serrano, J. J. (2016). Una aproximación al enfoque del capital social y a su contribución al estudio de los procesos de desarrollo local. Investigaciones Regionales - Journal of Regional Research, 34 (Otoño 2016), 49-71.

Febles, J. (2008). Los Modelos de Rasch en Administración de Empresas: Aplicaciones Avanzadas. Santa Cruz de Tenerife: Fundación FYDE Caja Canarias.

García, E. (2014). Políticas públicas para el desarrollo: efectividad, eficiencia y rentabilidad. Globalización y Desarrollo. ICE, Septiembre-Octubre 2014(880), 151-163.

Gaviria, M.A. (2013). Opciones de desarrollo local: el caso de un municipio risaraldense. Revista Académica e Institucional Paginas de la UCP, 94, 157 - 174.

Granados, H., Giraldo, O., \& Acevedo, N. (2016). Promoción de la competitividad y el desarrollo territorial en los municipios del Valle de Aburrá. Semestre Económico, 19(40), 93-116.

Gutiérrez, L.E. (2007). Potencial de Desarrollo y Gestión de la Política Regional. El Caso de Chihuahua. El Colegio de la Frontera Norte, 19(38), 7-35.

Linacre, J.M. (2014). WINSTEPS®. Rasch Measurement Computer Program User's Guide. Beaverton, Oregon: Winsteps. com. (Versión 3.81.0).

Lord, F.M. (1953). On the Statistical Treatment of Football Numbers. American Psychologist, 8, 750751.

Masters, G.N., \& Wright, B.D. (1984). The essential process in a family of measurement models. Psychometrika, 49, 529-544.

Michell, J. (1999). Measurement in psychology: a critical history of a methodological concept. Cambridge (UK): Cambridge University Press. 
Morán-Álvarez, J.C. \& Álvarez-Martínez, P. (2001). Medida del desarrollo humano para los países de la América Latina. El Trimestre Económico, 195-208.

Nunes, O.M., \& Karnopp, E. (2015). As Potencialidades Endógenas do Desenvolvimento Regional. Estudo de Caso do Município de Júlio de Castilhos/RS. Desenvolvimento em questao, Editora Unijuí, 13 (30), 203-229.

Oreja, J.R. (2005). Introducción a la medición objetiva en Economía, Administración y Dirección de Empresas: El Modelo de Rasch. IUDE. Serie Estudios, 2005/47. Santa Cruz de Tenerife: Instituto Universitario de la Empresa (IUDE) de la Universidad de La Laguna. Descargado de: http://iude.webs.ull.es/investigacion/publicaciones/pdf_docs_trabajo/iude-0547.pdf

Peña, A.R. (2006). Las disparidades económicas intrarregionales en Andalucía. Cádiz: Ed. Servicio de Publicaciones de la Universidad de Cádiz.

Peña, A.R. (2010). Los desequilibrios económicos territoriales: el caso de la provincia de Cádiz. Revista de Economía, XXVII(74), 9-47.

Perline, R., Wright, B.D., \& Wainer, H. (1979). The Rasch Model as an Additive Conjoint Measurement. Applied Psychological Measurement, 3(2), 237-255.

Prieto, G., \& Delgado, A.R. (2003). Análisis de un test mediante el modelo de Rasch. Psicothema, 15(1), 94-100.

Rasch, G. (1980). Probabilistic Models for Some Intelligence and Attainment Test. Chicago: The University Chicago Press.

Reig, E. (2010). Análisis del potencial socioeconómico de municipios rurales con métodos no paramétricos. Aplicación al caso de una zona Leader. Fundación BBVA. Documento de trabajo, $4 / 2010$.

Ruiz, R.G., \& Becerra, F.A. (2015). Una propuesta para la evaluación integral de los proyectos de desarrollo local. El caso de estudio TROPISUR. Economía y Desarrollo, 154(1), 144-154.

Sánchez, L., \& Blanco, B. (2012). El Modelo de Rasch en Dirección de Operaciones (Rasch Model in Operations Management). Working Papers on Operations Management, 3(2), 35-47.

Santinha, G. (2014). O princípio de coesão territorial enquanto novo paradigma de desenvolvimento na formulação de políticas públicas: (re)construindo ideias dominantes. Revista Latinoamericana de Estudios Urbano Regionales (EURE), 40(119), 75-97.

Stenner, A.J. (1994). Specific objectivity-local and general. Rasch Measurement Transactions, 8(3), 374.

Stimson, R.J. (2016). Some Challenges for Regional Science Research". En "New frontiers of regional and urban analysis. Investigaciones Regionales - Journal of Regional Research, 36, 11-34.

Varela, E. (2015). Nuevos roles de los gobiernos locales en la implementación de políticas públicas. Gobernabilidad territorial y competitividad global. Revista Latinoamericana de Estudios Urbano Regionales (EURE), 41(123), 211-237. 